\title{
Low-Level Updraft Intensification in Response to Environmental Wind Profiles $\mathscr{O}$
}

\author{
Nicholas A. Goldacker ${ }^{a}$ AND MATTHew D. PARKeR ${ }^{a}$ \\ ${ }^{a}$ Department of Marine, Earth, and Atmospheric Sciences, North Carolina State University, Raleigh, North Carolina
}

(Manuscript received 21 November 2020, in final form 29 March 2021)

\begin{abstract}
Supercell storms can develop a "dynamical response" whereby upward accelerations in the lower troposphere amplify as a result of rotationally induced pressure falls aloft. These upward accelerations likely modulate a supercell's ability to stretch near-surface vertical vorticity to achieve tornadogenesis. This study quantifies such a dynamical response as a function of environmental wind profiles commonly found near supercells. Self-organizing maps (SOMs) were used to identify recurring low-level wind profile patterns from 20194 model-analyzed, near-supercell soundings. The SOM nodes with larger $0-500 \mathrm{~m}$ storm-relative helicity $(\mathrm{SRH})$ and streamwise vorticity $\left(\omega_{\mathrm{s}}\right)$ corresponded to higher observed tornado probabilities. The distilled wind profiles from the SOMs were used to initialize idealized numerical simulations of updrafts. In environments with large $0-500 \mathrm{~m} \mathrm{SRH}$ and large $\omega_{\mathrm{s}}$, a rotationally induced pressure deficit, increased dynamic lifting, and a strengthened updraft resulted. The resulting upward-directed accelerations were an order of magnitude stronger than typical buoyant accelerations. At $500 \mathrm{~m}$ AGL, this dynamical response increased the vertical velocity by up to $25 \mathrm{~m} \mathrm{~s}^{-1}$, vertical vorticity by up to $0.2 \mathrm{~s}^{-1}$, and pressure deficit by up to $5 \mathrm{hPa}$. This response specifically augments the nearground updraft (the midlevel updraft properties are almost identical across the simulations). However, dynamical responses only occurred in environments where $0-500 \mathrm{~m} \mathrm{SRH}$ and $\omega_{\mathrm{s}}$ exceeded $110 \mathrm{~m}^{2} \mathrm{~s}^{-2}$ and $0.015 \mathrm{~s}^{-1}$, respectively. The presence versus absence of this dynamical response may explain why environments with higher $0-500 \mathrm{~m} \mathrm{SRH}$ and $\omega_{\mathrm{s}}$ correspond to greater tornado probabilities.
\end{abstract}

KEYWORDS: Convective-scale processes; Mesoscale processes; Tornadogenesis; Cloud resolving models; Mesoscale models; Supercells; Machine learning

\section{Introduction}

Despite most significant tornadoes (EF2+) occurring in supercell thunderstorms, not all supercells produce tornadoes; Trapp et al. (2005) found that perhaps only $\approx 25 \%$ of supercell mesocyclones are tornadic. The operational discrimination between nontornadic and tornadic supercells has proven difficult because they have similar kinematic flow properties, precipitation structures, and near-ground circulations (Blanchard and Straka 1998; Trapp 1999; Wakimoto and Cai 2000; Markowski et al. 2002, 2008, 2011; Klees et al. 2016). There have been some advances in identifying tornado environments (e.g., Thompson et al. 2003, 2007, 2012), but our incomplete understanding of environmental controls on the process nevertheless results in National Weather Service tornado warning false alarm rates near $75 \%$ in the United States (Brotzge et al. 2011; Anderson-Frey et al. 2016).

Attempts to predict the tornadic or nontornadic behavior of a specific supercell have centered on understanding the processes that govern supercell maturation and subsequent tornadogenesis. The process of supercell mesocyclonic tornadogenesis can be characterized by three steps (e.g., DaviesJones 2015): 1) the formation of a midlevel [e.g., $\approx 3-7 \mathrm{~km}$

Supplemental information related to this paper is available at the Journals Online website: https://doi.org/10.1175/JAS-D-200354.s1.

Corresponding author: Nicholas A. Goldacker, nagoldac@ ncsu.edu above ground level (AGL)] mesocyclone, 2) the generation of vertical vorticity $(\zeta)$ near the surface, and 3 ) the formation of a tornado via the contraction (convergence and stretching) of the resultant near-surface $\zeta$. It has long been understood that $\zeta$ (i.e., updraft rotation) in supercells originates from the updraft's tilting of ambient environmental horizontal vorticity, which is a consequence of environmental vertical wind shear (e.g., Rotunno and Klemp 1982; Davies-Jones 1984; Rotunno and Klemp 1985). The focus of tornadogenesis research has typically been on the less-understood origin of near-surface vorticity (step 2) in tornadic storms. Recent working hypotheses include the baroclinic generation and subsequent tilting of horizontal vorticity in association with a downdraft (e.g., Davies-Jones and Brooks 1993; Rotunno and Klemp 1985; Wicker and Wilhelmson 1995; Adlerman et al. 1999; Markowski et al. 2008, 2012; Dahl 2015; Parker and Dahl 2015) as well as the frictional generation and subsequent tilting of horizontal vorticity at the surface (Schenkman et al. 2014; Markowski 2016; Roberts et al. 2016; Roberts and Xue 2017; Roberts et al. 2020).

However, Coffer and Parker (2017) have demonstrated that both tornadic and nontornadic storms produce abundant pretornadic $\zeta$ at the surface. In other words, the details of step 2 alone do not appear to determine whether a supercell will form a tornado. This prompted them to focus on the vorticity contraction process in supercells in subsequent research. For the near-surface $\zeta$ to be stretched and amplified into a tornadic vortex (step 3 ), it is most favorable for the supercell to possess an overlying dynamically induced pressure gradient acceleration which can lift surface circulations upward even when the air is neutrally or negatively 
buoyant (e.g., Wicker and Wilhelmson 1995; Orf et al. 2017; Yokota et al. 2018).

Such an upward acceleration is often associated with rotationally induced pressure falls in the mesocyclone aloft (e.g., Rotunno and Klemp 1982; Weisman and Rotunno 2000). In turn, the base height and intensity of the mesocyclone is likely controlled by the vertical wind shear concentrated in the lowest $\sim 1 \mathrm{~km}$ AGL (e.g., Markowski et al. 2012; Markowski and Richardson 2014). Successive research has revealed that an increase in lower tropospheric streamwise horizontal vorticity $\left(\omega_{\mathrm{s}}\right)$ and storm-relative helicity (SRH) can dramatically increase the strength of the mesocyclone, associated dynamic lifting at low levels, and likelihood of tornadogenesis (e.g., Markowski and Richardson 2014; Skinner et al. 2014; Coffer and Parker 2015, 2017, 2018; Coffer et al. 2017; Flournoy et al. 2020). We refer to this process as the "dynamical response" in our study.

Coffer and Parker (2017) showed that trajectories within the low-level mesocyclone of their simulated supercells originated from below $300 \mathrm{~m}$ AGL in the environment. This led them to specifically focus on $\omega_{\mathrm{s}}$ and SRH in the 0-500 m AGL layer (and even shallower layers; e.g., Coffer et al. 2020) as indicators that the dynamical response might occur. Coffer and Parker (2017) also found that a wind profile with predominantly crosswise horizontal vorticity $\left(\omega_{\mathrm{c}}\right)$ near the ground caused more disorganized, unsteady low-level mesocyclones, which stunted the dynamical response. Similarly, Murdzek et al. (2020) determined that near-ground $\omega_{\mathrm{c}}$ precluded a favorable configuration of the mesocyclone for tornadogenesis in a nontornadic supercell observed by mobile radars.

Alongside the aforementioned process studies, it is well established from climatologies that the near-ground wind profile is meaningful for tornado forecasting (Rasmussen and Blanchard 1998; Markowski et al. 2003; Thompson et al. 2003; Esterheld and Giuliano 2008; Nowotarski and Jensen 2013; Coffer et al. 2019). Our working hypothesis is that parameters such as 0-500 m SRH have skill (Coffer et al. 2019) in particular due to the link connecting low-level SRH (and $\omega_{\mathrm{s}}$ ) to lowlevel mesocyclone strength, associated dynamic lifting, and thus completion of step 3 of tornadogenesis. We focus at present on the following unanswered questions:

1) Is there an identifiable subset of wind profiles over which the link connecting the low-level environmental winds, lowlevel dynamical response, and tornadogenesis probabilities is most prominent?

2) How much does the low-level dynamical response contribute to enhancements in low-level vertical velocity $(w)$ and low-level vertical vorticity $(\zeta)$ ?

Ultimately, for useful prediction of tornadoes, the answers to these questions need to be specific and quantitative. The goal of this study is to answer the above questions by linking the supercell low-level dynamical response to a spectrum of lower-tropospheric wind profiles. This study does not directly evaluate tornadogenesis but rather investigates how the lowlevel updraft evolves as it interacts with the low-level environmental wind profile. We accomplish this by examining 20194 near-storm proxy soundings composed of tornadic and nontornadic events spanning 12 years across the continental United States. A distillation of these soundings (obtained via the self-organizing map technique) is then used in an ensemble of idealized simulations to identify and quantify the range of dynamical responses. Details about the design of the study are presented in section 2, results are presented in section 3 , and a discussion of these results, experiment synthesis, and identification of future work are provided in section 4.

\section{Data and methods}

\section{a. Sounding data}

This study utilized near-storm proxy soundings from model analyses to depict right-moving supercell environments. The dataset is identical to that used by Coffer et al. (2019), which is an expansion of what was originally described by Smith et al. (2012) and Thompson et al. (2012). The soundings represent a collection of 20194 severe weather events from 2005 to 2017 for tornadic supercells and from 2005 to 2015 for nontornadic supercells (Coffer et al. 2019, their Fig. 1). Soundings through April 2012 are derived from the Rapid Update Cycle (RUC) model (Benjamin et al. 2004) while later soundings are derived from the Rapid Refresh (RAP) model (Benjamin et al. 2016). The model base-state profiles were combined with the Storm Prediction Center's mesoscale surface objective analysis (SFCOA; Bothwell et al. 2002) to produce a sounding that is composed of two parts: 1) surface data resulting from the SFCOA (which utilized available observations) and 2) RUC/RAP model data at pressure levels above the surface from the closest gridpoint to these 20194 severe weather events. The resulting soundings have data recorded on isobaric levels (25 hPa intervals). Because varying locations have different bottom isobaric levels, the profiles were interpolated to heights AGL from 0 to $12 \mathrm{~km}$ at $50 \mathrm{~m}$ intervals. The events were given further classifications of nontornadic (NT; 10839 cases), weakly tornadic [WT; (E)F0-1 tornadic damage, 7743 cases], and significantly tornadic [ST; (E)F2-5 tornadic damage, 1612 cases].

For this study, the primary focus was upon the resultant wind profiles, although some simple comparisons to thermodynamic parameters are also noted. Four variations of the heightinterpolated wind profiles were created: unaltered winds [ground relative (GR)], winds rotated to align the $0-6 \mathrm{~km}$ vector wind difference with the abscissa [ground-relative rotated (GRR)], winds converted to storm-relative winds [storm relative (SR)], and winds converted to storm relative and then rotated to align the $0-6 \mathrm{~km}$ vector wind difference with the abscissa [storm-relative rotated (SRR)]. The hodograph rotation process is similar to that used by Parker (2014), and the storm motions were approximated by the Bunkers supercell storm motion calculation (Bunkers et al. 2000). Hereafter, the GR, GRR, SR, and SRR sets of wind profiles will be referred to as "suites."

The use of four wind profile suites allowed us to isolate the impacts of hodograph shape versus prevailing wind direction and storm-relative flow. The GR wind suite captures impacts of prevailing wind speeds (which might be linked to season or 
TABLE 1. A summary of the settings and methods utilized in our self-organizing map process. The neighborhood function and learning rate asymptotically decrease toward zero as the number of iterations approaches infinity to allow for progressive refinement of the final 16 nodes. A $500 \mathrm{~m}$ depth was selected from a series of 12 tested depths (lowest $500 \mathrm{~m}$ to $6 \mathrm{~km}$ in $500 \mathrm{~m}$ increments, shown in Fig. S1) because this depth corresponded to a convergence of low topographic and quantization errors (TE and QE, respectively), which correlate to greater mapping quality (e.g., Liu et al. 2006; Ponmalai and Kamath 2019). A separate verification test (not shown) demonstrated convergence (as determined by a quasi-steady, asymptotic plateau in the quantization error) occurred around 8000 iterations.

\author{
Lattice design \\ Initialization method for reference nodes (weights) \\ Training data \\ Training method \\ Neighborhood function shape \\ Neighborhood function width \\ Learning rate (rate of convergence) \\ Iterations
}

$4 \times 4$ rectangular grid $(16$ nodes per $\mathrm{SOM})$

Principal component analysis

20194 0-500 m AGL zonal $(u)$ and meridional $(v)$ wind profiles

Random, seeded

Gaussian

0.24

0.10

10000 (one iteration per seeded, random selection of a $0-500 \mathrm{~m}(u, v)$ wind profile) synoptic regime). The nonrotated wind suites (GR and SR) capture impacts of prevailing wind directions (which might be linked to synoptic regime or geographic location). The predominant consideration for storm-scale dynamics is thought to be the shape of the storm-relative hodograph, and this is most purely isolated in the SRR suite.

\section{b. Self-organizing map technique}

As a baseline for our numerical modeling study, we seek an "executive summary" of the information contained in our 20194 profiles. Self-organizing maps (SOMs) represent an unsupervised learning process which results in a topographically ordered lattice (a spatial arrangement of nodes) that are tuned to various signals and features of the input dataset (e.g., Kohonen 1982, 1990, 1997). It has proven a useful tool for distilling large environmental sounding datasets (e.g., Nowotarski and Jensen 2013; Anderson-Frey et al. 2017; Nowotarski and Jones 2018).

We utilized the Python package MiniSom for this experiment (Vettigli 2019). We tested training of the SOMs on the $u$ and $v$ wind profiles over different depths, ranging from $0-500 \mathrm{~m}$ to $0-6 \mathrm{~km}$ AGL, increasing in $500 \mathrm{~m}$ increments. Via these trials, we found that the $0-500 \mathrm{~m}$ layer produced the lowest (most desirable) topographic error and quantization error (e.g., Liu et al. 2006; Ponmalai and Kamath 2019; Fig. S1 in the online supplemental materials); this also nicely corresponds to the layer emphasized by Coffer and Parker (2017) and Coffer et al. (2019), so it was adopted for our production SOMs. We used 16 output nodes for each suite of wind profiles to create an optimal balance between resolving dataset features and avoiding repetition across the final nodes. Other SOM attributes for our study are summarized in Table 1.

After the final iteration of the SOM process, the 20194 wind profiles were assigned to a final best-matching node. All members of each node were then averaged to yield 16 output profiles (one per node). These averages represent the distillation of recurring structures in the set of 20194 events for each suite. The GR, GRR, SR, and SRR wind profile variations were created before the SOM algorithm was utilized thereby resulting in different SOM lattices (nodes), nodal constituents (i.e., wind profiles in each node), node-averaged wind profiles, and computed nodal parameters (e.g., SRH, $\omega_{\mathrm{s}}$, and $\omega_{\mathrm{c}}$ ) for each suite. As initially stated, our goal was to create an "executive summary" representing the full span of supercell environments. Inspection of the four SOMs revealed that the GR and SRR suites had a greater diversity in nodes (a large range in values (from high to low) of \%ST and \%NT in addition to a larger variety of node-averaged wind profiles) than the other suites. Therefore, these two suites were selected for detailed study. In section $3 \mathrm{a}$, we provide some basic interpretation of the information in these SOMs, although their primary purpose is to initialize our numerical modeling study. The averaged wind profiles from the SOM nodes were taken as input environments for the numerical experiment described next.

\section{c. Model configuration}

Idealized simulations were performed using version 19 of the Bryan Cloud Model 1 (CM1) from Bryan and Fritsch (2002). The spatial extent of the domain was $81.6 \mathrm{~km} \times$ $81.6 \mathrm{~km} \times 15 \mathrm{~km}$ with a horizontal grid spacing of $100 \mathrm{~m}$ and a vertical grid spacing that stretched from $50 \mathrm{~m}$ near the surface to $100 \mathrm{~m}$ at $15 \mathrm{~km}$. Coriolis accelerations, radiation, and surface fluxes were neglected. The mean wind profiles from each of the SOM nodes (16 each from the GR and SRR suites) served as homogeneous initial conditions. The winds from our SOM nodes were utilized up through $12 \mathrm{~km}$ AGL, above which the winds were held constant throughout an emulated stratosphere.

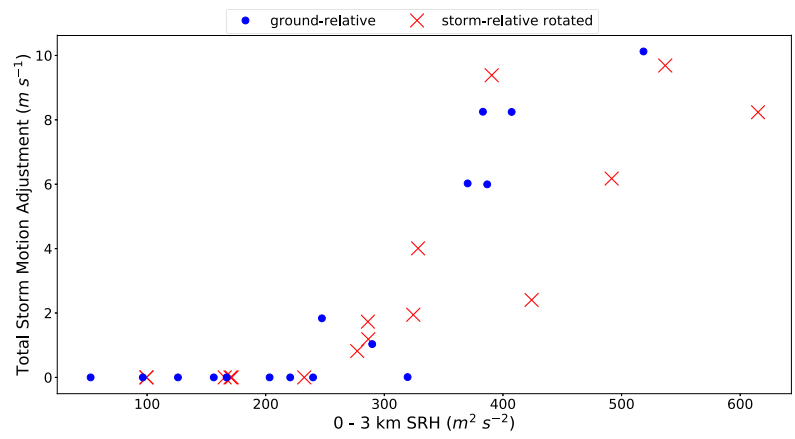

FIG. 1. A scatterplot demonstrating the relationship between $0-3 \mathrm{~km} \mathrm{SRH}\left(\mathrm{m}^{2} \mathrm{~s}^{-2}\right)$ and the required adjustment to Bunkers storm motions. 
TABLE 2. A summary of the ground-relative (GR) and storm-relative rotated (SRR) environments' 0-500 m shear parameters SRH $\left(\mathrm{m}^{2} \mathrm{~s}^{-2}\right), \omega_{\mathrm{s}}\left(\mathrm{s}^{-1}\right)$, and $\omega_{\mathrm{c}}\left(\mathrm{s}^{-1}\right)$ as impacted by storm motion. The variations utilizing the initial Bunkers storm motions ("b") and the final domain translation motions ("d") along with their differences ("d" - "b") are included. The values reported in the text use the domain motion.

\begin{tabular}{|c|c|c|c|c|c|c|c|c|c|}
\hline & $\mathrm{SRH}_{\mathrm{b}}$ & $\mathrm{SRH}_{\mathrm{d}}$ & $\Delta \mathrm{SRH}$ & $\omega_{\mathrm{sb}}$ & $\omega_{\mathrm{sd}}$ & $\Delta \omega_{\mathrm{s}}$ & $\omega_{\mathrm{cb}}$ & $\omega_{\mathrm{cd}}$ & $\Delta \omega_{\mathrm{c}}$ \\
\hline \multicolumn{10}{|c|}{ GR environment } \\
\hline A & 63.0 & 63.0 & 0.0 & 0.0111 & 0.0111 & 0.0000 & -0.0051 & -0.0051 & 0.0000 \\
\hline $\mathrm{B}$ & 161.2 & 145.2 & -16.0 & 0.0222 & 0.0225 & 0.0003 & -0.0077 & -0.0067 & 0.0010 \\
\hline $\mathrm{C}$ & 252.2 & 161.8 & -90.4 & 0.0290 & 0.0296 & 0.0006 & -0.0074 & 0.0006 & 0.0080 \\
\hline $\mathrm{D}$ & 317.6 & 197.4 & -120.2 & 0.0331 & 0.0311 & -0.0020 & -0.0028 & 0.0109 & 0.0137 \\
\hline $\mathrm{E}$ & -6.7 & -6.7 & 0.0 & -0.0015 & -0.0015 & 0.0000 & -0.0064 & -0.0064 & 0.0000 \\
\hline $\mathrm{F}$ & 80.6 & 80.6 & 0.0 & 0.0131 & 0.0131 & 0.0000 & -0.0001 & -0.0001 & 0.0000 \\
\hline G & 174.5 & 164.4 & -10.1 & 0.0230 & 0.0231 & 0.0001 & -0.0023 & -0.0018 & 0.0005 \\
\hline $\mathrm{H}$ & 238.0 & 151.4 & -86.6 & 0.0282 & 0.0253 & -0.0029 & 0.0014 & 0.0124 & 0.0110 \\
\hline I & -0.9 & -1.0 & -0.1 & -0.0016 & -0.0016 & 0.0000 & 0.0090 & 0.0090 & 0.0000 \\
\hline $\mathrm{J}$ & 23.8 & 23.8 & 0.0 & 0.0043 & 0.0043 & 0.0000 & -0.0003 & -0.0003 & 0.0000 \\
\hline $\mathrm{K}$ & 114.0 & 114.0 & 0.0 & 0.0170 & 0.0170 & 0.0000 & 0.0049 & 0.0049 & 0.0000 \\
\hline $\mathrm{L}$ & 198.6 & 136.4 & -62.2 & 0.0247 & 0.0219 & -0.0028 & 0.0058 & 0.0129 & 0.0071 \\
\hline M & 96.1 & 96.1 & 0.0 & 0.0120 & 0.0120 & 0.0000 & 0.0130 & 0.0130 & 0.0000 \\
\hline $\mathrm{N}$ & 25.5 & 25.5 & 0.0 & 0.0035 & 0.0035 & 0.0000 & 0.0044 & 0.0044 & 0.0000 \\
\hline $\mathrm{O}$ & 76.0 & 76.0 & 0.0 & 0.0108 & 0.0108 & 0.0000 & 0.0061 & 0.0061 & 0.0000 \\
\hline $\mathrm{P}$ & 174.7 & 112.2 & -62.5 & 0.0217 & 0.0176 & -0.0041 & 0.0101 & 0.0164 & 0.0063 \\
\hline \multicolumn{10}{|c|}{ SRR environment } \\
\hline A & 74.2 & 64.3 & -9.9 & 0.0064 & 0.0057 & -0.0007 & 0.0066 & 0.0072 & 0.0006 \\
\hline $\mathrm{B}$ & 79.4 & 74.1 & -5.3 & 0.0088 & 0.0088 & 0.0000 & 0.0047 & 0.0047 & 0.0000 \\
\hline $\mathrm{C}$ & 246.9 & 182.1 & -64.8 & 0.0239 & 0.0242 & 0.0003 & -0.0024 & 0.0000 & 0.0024 \\
\hline $\mathrm{D}$ & 292.9 & 209.5 & -83.4 & 0.0231 & 0.0228 & -0.0003 & 0.0024 & 0.0062 & 0.0038 \\
\hline $\mathrm{E}$ & 25.0 & 25.0 & 0.0 & 0.0028 & 0.0028 & 0.0000 & 0.0031 & 0.0031 & 0.0000 \\
\hline $\mathrm{F}$ & 134.6 & 121.6 & -13.0 & 0.0170 & 0.0171 & 0.0001 & -0.0029 & -0.0024 & 0.0005 \\
\hline $\mathrm{G}$ & 180.3 & 146.0 & -34.3 & 0.0193 & 0.0194 & 0.0001 & -0.0010 & 0.0000 & 0.0010 \\
\hline $\mathrm{H}$ & 350.8 & 229.3 & -121.5 & 0.0304 & 0.0311 & 0.0007 & -0.0048 & 0.0007 & 0.0055 \\
\hline I & 26.7 & 26.7 & 0.0 & 0.0055 & 0.0055 & 0.0000 & 0.0003 & 0.0003 & 0.0000 \\
\hline $\mathrm{J}$ & 43.0 & 43.0 & 0.0 & 0.0067 & 0.0067 & 0.0000 & 0.0015 & 0.0015 & 0.0000 \\
\hline $\mathrm{K}$ & 205.4 & 184.3 & -21.1 & 0.0267 & 0.0268 & 0.0001 & -0.0045 & -0.0032 & 0.0013 \\
\hline $\mathrm{L}$ & 265.0 & 156.7 & -108.3 & 0.0276 & 0.0275 & -0.0001 & -0.0024 & 0.0041 & 0.0065 \\
\hline M & 60.5 & 60.5 & 0.0 & 0.0131 & 0.0131 & 0.0000 & 0.0055 & 0.0055 & 0.0000 \\
\hline $\mathrm{N}$ & 90.4 & 90.4 & 0.0 & 0.0151 & 0.0151 & 0.0000 & 0.0036 & 0.0036 & 0.0000 \\
\hline $\mathrm{O}$ & 65.7 & 65.7 & 0.0 & 0.0094 & 0.0094 & 0.0000 & 0.0017 & 0.0017 & 0.0000 \\
\hline $\mathrm{P}$ & 189.8 & 181.2 & -8.6 & 0.0238 & 0.0238 & 0.0000 & 0.0020 & 0.0023 & 0.0003 \\
\hline
\end{tabular}

To isolate the sensitivities of an updraft and low-level mesocyclone to the low-level wind profile, the simulations used an imposed artificial vertical velocity tendency ( $w$-tendency, $\dot{w}$ ) within a controlled thermal profile. The scales and structure of the tendency were adjusted by trial and error until they produced updrafts with the same sizes and magnitudes as those in Coffer and Parker (2017, 2018), Coffer et al. (2017), and Parker (2017). Here, $\dot{w}$ was applied in an ellipsoid with a peak amplitude forcing of $0.49 \mathrm{~m} \mathrm{~s}^{-2}$ that varied as the squared cosine of distance from its center at $5.25 \mathrm{~km}$ above the grid origin, falling to 0 at vertical and horizontal radii of 4.75 and $3 \mathrm{~km}$, respectively. A cross section of the vertical velocity tendency is provided in Fig. S2 in the online supplemental materials. Given this structure, the averaged tendency within the ellipsoid was $0.15 \mathrm{~m} \mathrm{~s}^{-2}$, roughly equivalent to the buoyancy associated with a $4.5 \mathrm{~K}$ temperature excess. The lowest height where a nonzero $\dot{w}$ exists is $500 \mathrm{~m}$ AGL, which can be thought of as an effective level of free convection (LFC) for the simulated updraft. The tendency was applied constantly for the full duration of the simulations (45 $\mathrm{min})$. Analyses were not conducted beyond $45 \mathrm{~min}$ because, consistent with right-moving supercells that evolve in nature, the stronger simulated updrafts acquired deviant motion and propagated away from the artificial forcing centroid. As a result, undesired, unnatural feedbacks developed and contaminated the organic interaction of the idealized updraft with the wind profile.

By design, the use of $\dot{w}$ produces identical baseline updrafts across all experiments, and we use thermodynamic profiles that prohibit other sources of buoyancy from developing and modifying the resulting updraft. All simulations used a dry adiabatic "troposphere" (having $300 \mathrm{~K}$ potential temperature and $1000 \mathrm{hPa}$ surface pressure) from 0 to $12 \mathrm{~km}$, with a stratospheric potential temperature lapse rate of $20 \mathrm{~K} \mathrm{~km}^{-1}$ extending from $12 \mathrm{~km}$ to the model top. No moisture was included. The use of a dry adiabatic lapse rate (i.e., neutral static stability) in conjunction with large vertical wind shear near the 


\section{Ground-relative}

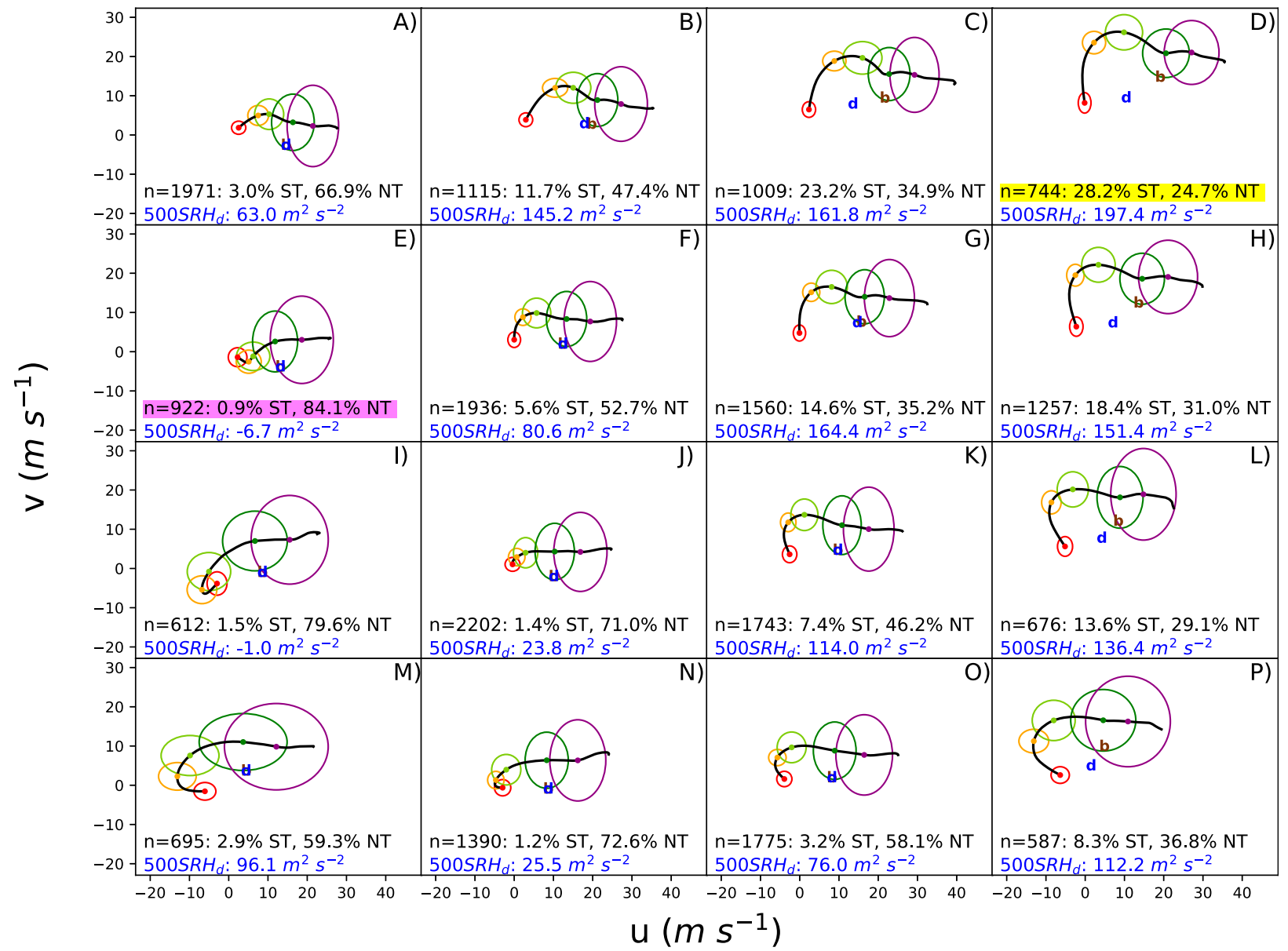

FIG. 2. The resultant SOM for the ground-relative (GR) suite of wind profiles. The black line is the average wind profile from all of the input profiles in that node from 0 to $12 \mathrm{~km}, n$ is the number of samples, ST is the percentage of significantly tornadic cases, and NT is the percentage of nontornadic cases. Note the true sum of the ST, WT (percentage of weakly tornadic cases, not shown), and NT cases is $100 \%$; however, due to rounding, the presented percentages may not equal $100 \%$. The node with the largest $\%$ ST ( $\%$ NT) is highlighted in yellow (pink). The ellipses are centered at $0 \mathrm{~km}$ (red), $0.5 \mathrm{~km}$ (orange), $1 \mathrm{~km}$ (yellow-green), $3 \mathrm{~km}$ (green), and $6 \mathrm{~km}$ (purple), and the radii are equivalent to twice the standard deviation (95\%) of the profiles in each node at those points. The center of the symbols "b" (brown) and "d" (blue) denote the initial Bunkers motion and final domain translation motion (effective storm motion), respectively. The parameter $500 \mathrm{SRH}_{\mathrm{d}}$ (blue, $\mathrm{m}^{2} \mathrm{~s}^{-2}$ ) is the $0-500 \mathrm{~m}$ SRH calculated using "d."

surface in some profiles led to the release of Kelvin-Helmholtz instability. To reduce the cascading effects resulting from such eddies and attendant numerical noise, artificial sixth-order diffusion was used (with a nondimensional coefficient of 0.08 ). On balance, the advantages of this configuration (a closely controlled experiment) outweighed the drawbacks.

As a first attempt, $\dot{w}$ was assumed to move at the mean storm motion given by the Bunkers et al. (2000) estimate for a given SOM node. In practice, $\dot{w}$ is fixed at the domain's center and the domain itself is translated with the Bunkers vector (thus, the terms "storm motion" and "domain motion" can be used interchangeably in these numerical experiments). In nature (and full physics simulations), supercells split, acquire deviant motion, and also interact with their evolving precipitation fields. The Bunkers storm motion empirically represents such effects on supercell motion, but it does so imperfectly (e.g., Bunkers et al. 2000, 2006; Zeitler and Bunkers 2005; Bunkers 2018). In particular, the present simulations were void of moist processes and conducted on the updraft scale; thus, they were not always perfectly suited to use of the Bunkers motion estimate. Our compensation for this incompatibility was as follows.

The present goal is to study how the wind profile impacts dynamical evolution of supercell-like updrafts in a simpler, more controlled setting than a real-world environment. It was therefore important 1) to ensure that low-level air near the surface actually flows into the simulated updrafts and ascends and 2) to keep the resulting centers of the simulated updrafts collocated with the center of $\dot{w}$. These ideals in some cases required slight adjustment of the implied storm motion (i.e., 


\section{Storm-relative rotated}

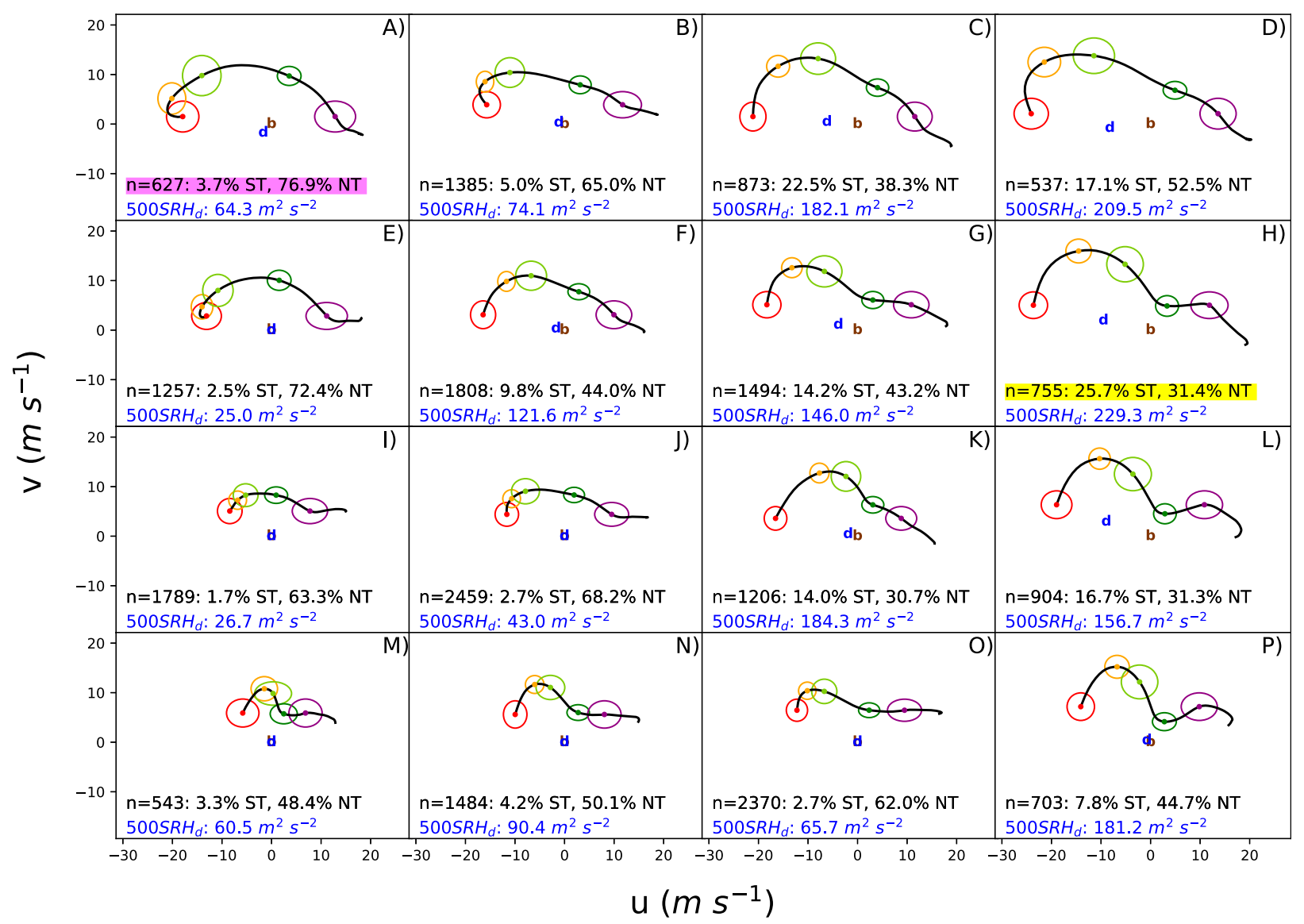

FIG. 3. As in Fig. 2, but for the storm-relative rotated (SRR) suite.

flow relative to the zone of $\dot{w})$ to ensure near-ground air $(0$ $1.5 \mathrm{~km}$ ) flowed into the simulated midlevel updrafts. Storm motions across the nodes were adjusted so that surface storm-relative flow (SRF) vectors greater than $15 \mathrm{~m} \mathrm{~s}^{-1}$ were reduced in magnitude to $15 \mathrm{~m} \mathrm{~s}^{-1}$ without changing the SRF direction. Then, for storms that initially propagated away from the $w$-tendency, the domain translation motion (effective storm motion) was modified as necessary to keep the $1-3 \mathrm{~km}$ segment of the updraft centered on $\dot{w}$ for the 45 simulated minutes. Domain translation adjustments were not needed for lower-SRH cases and generally increased with SRH for the higher-SRH cases (Fig. 1). This is in-line with recent findings by Bunkers (2018), who demonstrated that environments in the top 10 percentile of $0-3 \mathrm{~km} \mathrm{SRH}$ typically have the greatest Bunkers storm motion errors (with RMSE of $5 \mathrm{~m} \mathrm{~s}^{-1}$ and individual case departures well over $10 \mathrm{~m} \mathrm{~s}^{-1}$ ).

Although the nodal values of storm-motion dependent parameters (e.g., SRH) change as a result of these adjustments, they do not change which nodes have the most averaged SRH (Table 2), nor which have the most $\omega_{\mathrm{s}}$. In general, the effect of the adjustments is to decrease the SRH of the highest nodes (Table 2), which means that our experiments, if anything, understate the impacts of the differences in SRH found among the SOM nodes.

\section{d. Analysis techniques}

To analyze the evolution of the simulated updrafts in response to variations in the low-level environmental wind

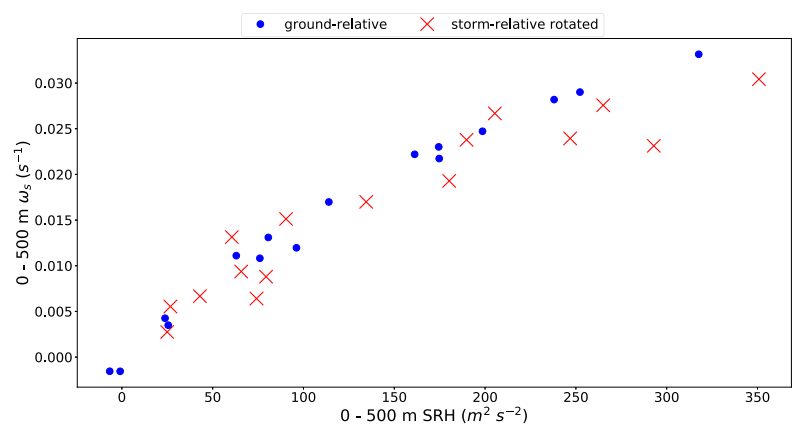

FIG. 4. A scatterplot demonstrating the relationship between $0-500 \mathrm{~m} \mathrm{SRH}\left(\mathrm{m}^{2} \mathrm{~s}^{-2}\right)$ and 0-500 $\mathrm{m}$ streamwise vorticity $\left(\omega_{\mathrm{s}}, \mathrm{s}^{-1}\right)$ for ground-relative (blue) and storm-relative rotated (red) SOM environments utilizing the initial Bunkers storm motion estimates. 

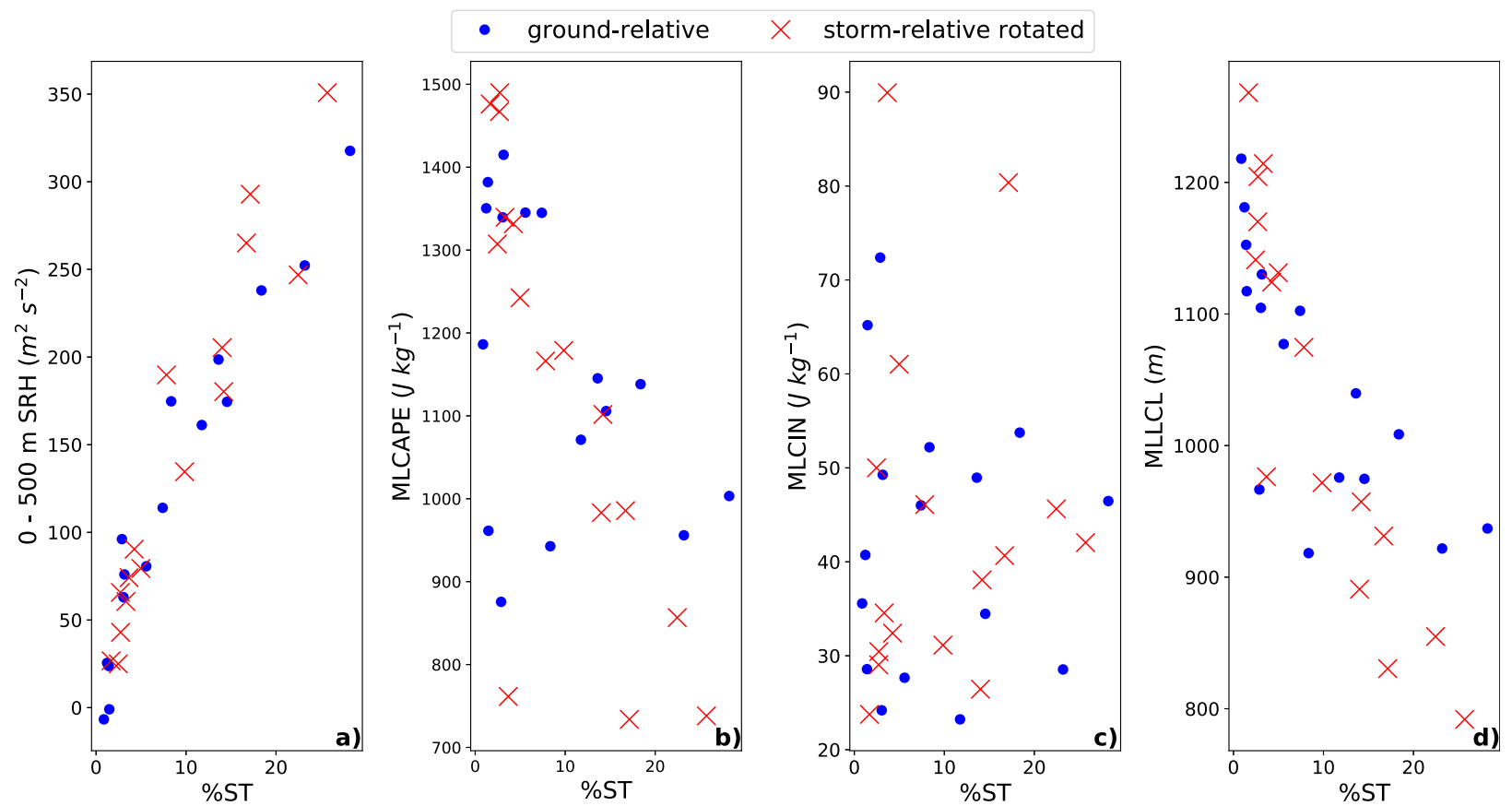

FIG. 5. Scatterplots depicting the relationships between the percentage of significantly tornadic cases (\%ST) and (a) 0-500 $\mathrm{m} \mathrm{SRH}$ $\left(\mathrm{m}^{2} \mathrm{~s}^{-2}\right)$, (b) MLCAPE $\left(\mathrm{J} \mathrm{kg}^{-1}\right)$, (c) MLCIN $\left(\mathrm{J} \mathrm{kg}^{-1}\right)$, and (d) MLLCL ( $\mathrm{m}$ ) for the ground-relative (blue) and storm-relative rotated (red) suites. The SRH values are calculated using the initial Bunkers storm motion estimates.

profiles, we utilize a combination of analyses, including pressure perturbation $\left(p^{\prime}\right)$ field decompositions and their associated vertical perturbation pressure gradient acceleration (VPPGA) decompositions. The framework utilized follows Wilhelmson and Ogura (1972), Rotunno and Klemp (1982), and Klemp (1987), in that we decompose the $p^{\prime}$ field produced by CM1 in each simulation into buoyant $\left(p_{\mathrm{B}}^{\prime}\right)$, dynamic linear $\left(p_{\mathrm{DL}}^{\prime}\right)$, and dynamic nonlinear $\left(p_{\mathrm{DNL}}^{\prime}\right)$ components. The iterative solver for the $p^{\prime}$ components is identical to that of Parker and Johnson (2004a,b), Parker (2007, 2010, 2017), Davenport and Parker (2015), and Coffer and Parker (2015, 2017). In this study, we expect the low-level dynamical response to emerge in $p_{\mathrm{DNL}}^{\prime}$, which is primarily associated with local rotation (low pressure) or deformation (high pressure) in the storm's perturbation wind field. The collection of VPPGAs generated by their respective perturbation pressure counterparts in conjunction with buoyancy $(B)$ and our artificial forcing $(\dot{w})$ comprises the vertical accelerations within our emulated updrafts:

$$
\frac{D w}{D t}=\dot{w}+\underbrace{B-\frac{1}{\rho_{\mathrm{o}}} \frac{\partial p_{\mathrm{B}}^{\prime}}{\partial z}}_{\mathrm{ACCB}} \underbrace{-\frac{1}{\rho_{\mathrm{o}}} \frac{\partial p_{\mathrm{DL}}^{\prime}}{\partial z}}_{\mathrm{VPPGAB}} \underbrace{-\frac{1}{\rho_{\mathrm{o}}} \frac{\partial p_{\mathrm{DNL}}^{\prime}}{\partial z}}_{\mathrm{VPPGADL}} .
$$

The second and third terms on the right-hand side of Eq. (1) yield the total buoyant acceleration (ACCB $=B+$ VPPGAB) and the last two terms on the right-hand side yield the total dynamic acceleration $(\mathrm{ACCD}=\mathrm{VPPGADL}+$ VPPGADNL). The rotation-associated VPPGADNL explains the dynamic lifting associated with the low-level dynamical response described in section 1 .

\section{Self-organizing map and idealized modeling results}

\section{a. Characteristics of self-organizing maps}

As described in section 2, four SOMs were created. Here we briefly summarize the structures of the GR (Fig. 2) and SRR (Fig. 3) suites because of their greater diversity among nodeaveraged wind profile patterns (as compared to the GRR and SR suites); interested readers can view SOMs for the GRR and SR suites in the online supplemental materials (Figs. S3 and S4, respectively). There exist prevalent trends across the SOMs for 0-500 m SRH (Fig. 2 and Fig. 3 for GR and SRR suites, respectively), which is expected since the $0-500 \mathrm{~m}(u, v)$ winds were used for training the SOMs. Similar trends also exist in $\omega_{\mathrm{s}}$ (not plotted in Figs. 2 and 3 but reported in Table 2). Not surprisingly, since SRH is the integrated storm-relative flux of $\omega_{\mathrm{s}}$, they are strongly related to one another in our dataset (Fig. 4). Hereafter, we will primarily mention 0-500 $\mathrm{m} \mathrm{SRH}$ to characterize the low-level wind profile.

The SOMs also effectively sorted the highest percentage of significantly tornadic cases (\%ST) into the highest SRH nodes (Fig. 5a). This was by no means guaranteed, as the SOM training excluded such information (it used only the $0-500 \mathrm{~m}$ wind profiles). The strong coupling of $0-500 \mathrm{~m} \mathrm{SRH}$ to $\% \mathrm{ST}$ provides independent confirmation that the low-level wind profile has strong influence on the tornadogenesis process (as reviewed in section 1 ). To assess the degree to which this signal 

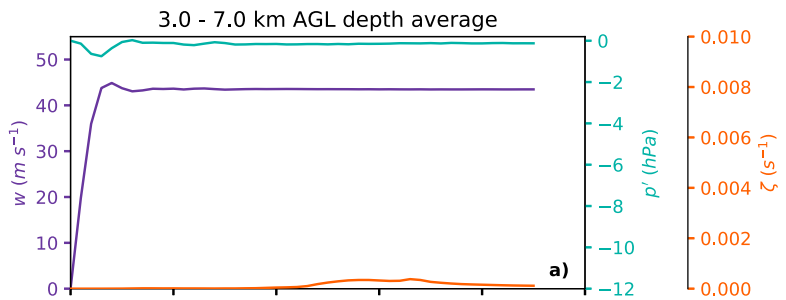

$1.0-3.0 \mathrm{~km}$ AGL depth average
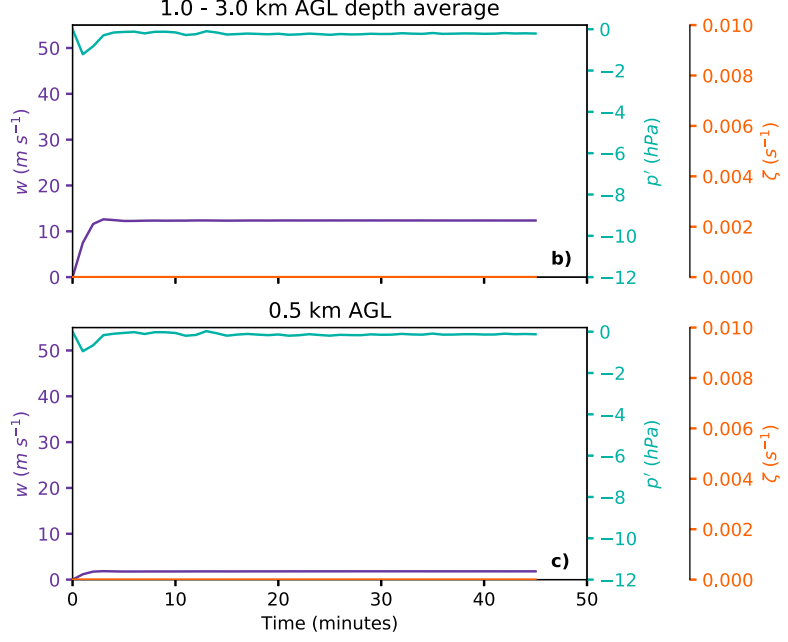

FIG. 6. Time series of $\dot{w}$ evolution in the quiescent environment (base state $u$ and $v$ winds are zero throughout the vertical profile). The depicted fields are maximum vertical velocity $\left(w\right.$, indigo, $\left.\mathrm{m} \mathrm{s}^{-1}\right)$, minimum pressure perturbation $\left(p^{\prime}\right.$, teal, $\left.\mathrm{hPa}\right)$, and maximum vertical vorticity ( $\zeta$, orange, $\mathrm{s}^{-1}$ ), computed (a) for the 3-7 km AGL layer average, (b) for the 1-3 km AGL layer average, and (c) locally at $0.5 \mathrm{~km}$ AGL. Fields are plotted at 1-min intervals and represent peak magnitudes (after vertical averaging) within a $4 \mathrm{~km} \times 4 \mathrm{~km}$ horizontal area centered on the artificial $w$-tendency $(\dot{w})$.

is solely attributable to the wind profile, we briefly examine whether any other environmental properties also covary with $\% \mathrm{ST}$. There are weak inverse relationships between \%ST and mixed-layer (ML) convective available potential energy (CAPE), convective inhibition (CIN), and lifting condensation level (LCL) (Figs. 5b,c,d, respectively). To an extent, these linkages occur because the higher shear (higher SRH) environments tend to occur in slightly cooler and moister settings (not shown). All of the thermodynamic variables' relationships (whether direct or inverse) to \%ST are weaker than that of \% ST to SRH itself, and the range of thermodynamic parameter values here is small in the context of typical values. But, the most distinctive thermodynamic signal, whereby lower LCLs are associated with higher probabilities of tornadogenesis, is certainly consistent with the findings of Rasmussen and Blanchard (1998) and Markowski et al. (2002).

We also found that location, hour, and season of storms' occurrence were not strongly linked to \%ST values of the SOM nodes (these distributions are provided in the online supplemental materials as Figs. S5, S6, and S7, respectively). Although the signals in the GR and SRR suites are similar (i.e., Figs. 2-5), the GR suite encompasses a wider range of \% ST among nodes $(0.9 \%-28.2 \%$ vs $1.7 \%-25.7 \%$ for the GR

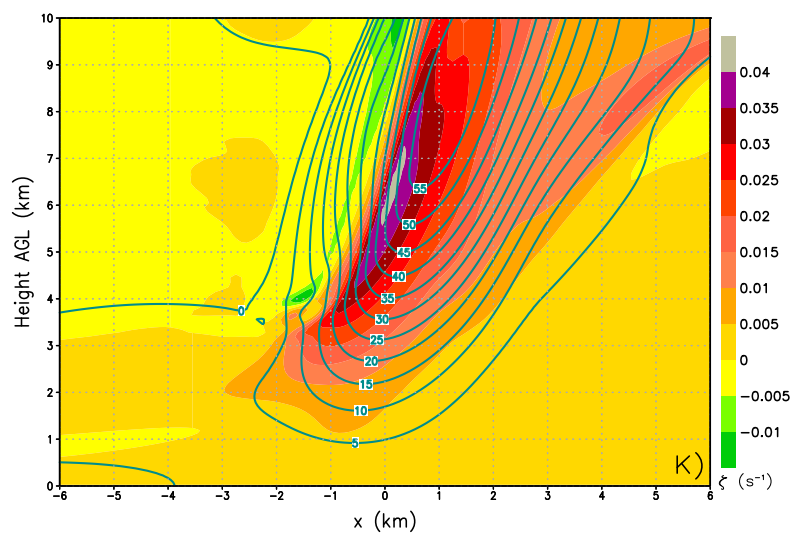

FIG. 7. A vertical $(x-z)$ cross section depicting the $2 \mathrm{D}$ vertical structure of the vertical velocity $(w)$ resulting from the vertical velocity tendency $(\dot{w})$ evolving in GR environment $\mathrm{K}$. Vertical velocity $(w)$ contours are given for every $5 \mathrm{~m} \mathrm{~s}^{-1}$ in teal, and vertical vorticity $(\zeta)$ is shaded $\left(\mathrm{s}^{-1}\right)$ in warm colors. These values are time averaged during the last $10 \mathrm{~min}$ of the 45 -min simulation.

and SRR suites, respectively). So, for brevity, we primarily focus on the GR suite for the remainder of this article (with occasional reference to the SRR suite for comparison). Numerical references to SRH, $\omega_{\mathrm{s}}$, or $\omega_{\mathrm{c}}$ from this point forward will refer to values computed using the actual domain motions in the experiments (not the original Bunkers estimates).

\section{b. Control experiment}

The SOMs provided a lattice of wind profile patterns representing the spectrum found in this dataset. Our working hypothesis is that these varying profiles may be determinative of an updraft's potential to exhibit the supercell low-level dynamical response. To achieve this goal, we analyze the ensemble of simulations initialized with the nodal wind profiles from the SOMs, with a focus on the $w, \zeta$, and $p^{\prime}$ evolutions. To isolate, evaluate, and quantify the supercell low-level dynamical response, three layers are analyzed in detail: 1) the layer typically containing a midlevel mesocyclone (3-7 km AGL), 2) the layer typically containing a low-level mesocyclone $(1-3 \mathrm{~km}$ AGL), and 3) the base of $\dot{w}$ which is our effective LFC (500 m AGL). When presented in time series form, all such analyses utilize maximum or minimum values (denoted in figure captions) computed in a $4 \mathrm{~km} \times 4 \mathrm{~km}$ area centered on the imposed $\dot{w}$.

To establish a benchmark for the behaviors in our SOM wind profiles, we first present the evolution of the idealized updraft that occurs in a quiescent environmental wind profile (referred to as the "control run"). In the calm environment, there is an initial impulse from the artificial forcing, after which the $\zeta, p^{\prime}$, and $w$ fields quickly reach a quasi-steady state for the remainder of the simulation (Fig. 6). In particular, $\zeta$ and $p^{\prime}$ remain nearly zero across all three of the aforementioned layers through the entire control run. The depth-averaged $w$ across the 3-7 km AGL layer reaches a steady-state value near $45 \mathrm{~m} \mathrm{~s}^{-1}$ (Fig. 6a). Across the 1-3 km AGL layer, the depthaveraged $w$ reaches a steady-state value near $12 \mathrm{~m} \mathrm{~s}^{-1}$ (Fig. 6b). At the $0.5 \mathrm{~km}$ AGL level, the $w$ asymptotically approaches $2 \mathrm{~m} \mathrm{~s}^{-1}$ (Fig. 6c). So, in a quiescent setting, the updraft forcing 


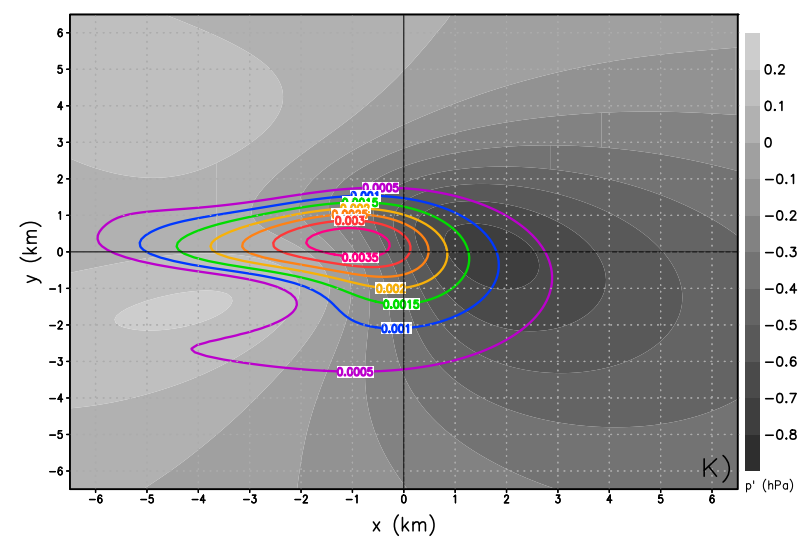

FIG. 8. A horizontal $(x-y)$ cross section at $0.5 \mathrm{~km}$ AGL depicting the $2 \mathrm{D}$ structure of the vertical velocity $(w)$ resulting from the vertical velocity tendency $(\dot{w})$ evolving in GR environment K. Vertical vorticity $(\zeta)$ contours are given for every $0.0005 \mathrm{~s}^{-1}$ (rainbow), and pressure perturbations are shaded (gray, hPa). These values are time averaged during the last $10 \mathrm{~min}$ of the $45-\mathrm{min}$ simulation.

is responsible for a baseline of $2 \mathrm{~m} \mathrm{~s}^{-1}$ ascent at $500 \mathrm{~m}$ AGL. Any low-level updraft intensification beyond this would represent the effect of a particular environment's wind profile. Indicators of a low-level dynamical response important to tornadogenesis would include increases in vertical vorticity nearly simultaneous with attendant decreases in pressure perturbation, closely followed by increases in vertical velocity at the lowest levels (e.g., at the effective LFC of $500 \mathrm{~m}$ AGL). This would indicate increased updraft rotation rate, associated dynamical lowering of the pressure (i.e., $p_{\mathrm{DNL}}^{\prime}$ ), and enhanced lifting from below the pressure minimum (i.e., VPPGADNL). The growth in $500 \mathrm{~m}$ AGL $\zeta, p^{\prime}$, and $w$ characteristic of a dynamical response is clearly absent in the quiescent control run (Fig. 6).

\section{c. Wind profile sensitivity simulations}

To demonstrate that the design of our artificial forcing is fostering flow resembling supercell updrafts, we present cross sections of the evolving flow in GR environment $\mathrm{K}$ (which was near the population median in $0-500 \mathrm{~m} \mathrm{SRH}$ ) during the final $10 \mathrm{~min}$ of the simulation. The simulated updraft has midlevel $w$ values comparable to the control run, but with a characteristic downshear tilt (Fig. 7). A collocated column of positive $\zeta$ emerges due to the tilting of ambient environmental vorticity (Fig. 7). In plan view, a kidney-shaped pressure minimum occurs slightly downshear of the center of updraft rotation (Fig. 8). This is characteristic of a supercell-like updraft, combining $p_{\mathrm{DNL}}^{\prime}$ which is minimized at the center of rotation with $p_{\mathrm{DL}}^{\prime}$ (the "updraft-in-shear effect") which is minimized on the updraft's downshear side. An animation of this basic updraft evolution is included in the online supplemental materials (Fig. S8).

As explained earlier, we focus our primary interpretation on the sensitivity tests using the GR hodograph suite (Fig. 2). For the midlevels (3-7 km), Fig. 9 shows that, after the initial impulsive response to the imposed $w$-tendency, there is no substantial further increase in $\zeta$, decrease in $p^{\prime}$, or increase in $w$. Across all environments, the midlevel response is a similar quasi-steady rotating updraft with $w$ of $40-45 \mathrm{~m} \mathrm{~s}^{-1}$ and $\zeta$ of $0.02-0.03 \mathrm{~s}^{-1}$. This is perhaps unsurprising since the wind profiles originated from only right-moving supercell environments. This uniformity among midlevel structures was not sensitive to the changes in the height or depth chosen to define this middle tropospheric layer (not shown). In short, our updraft forcing $(\dot{w})$ is producing nearly identical supercell-like updrafts in each simulation; differences attributable to the

3.0 - $7.0 \mathrm{~km}$ AGL depth average

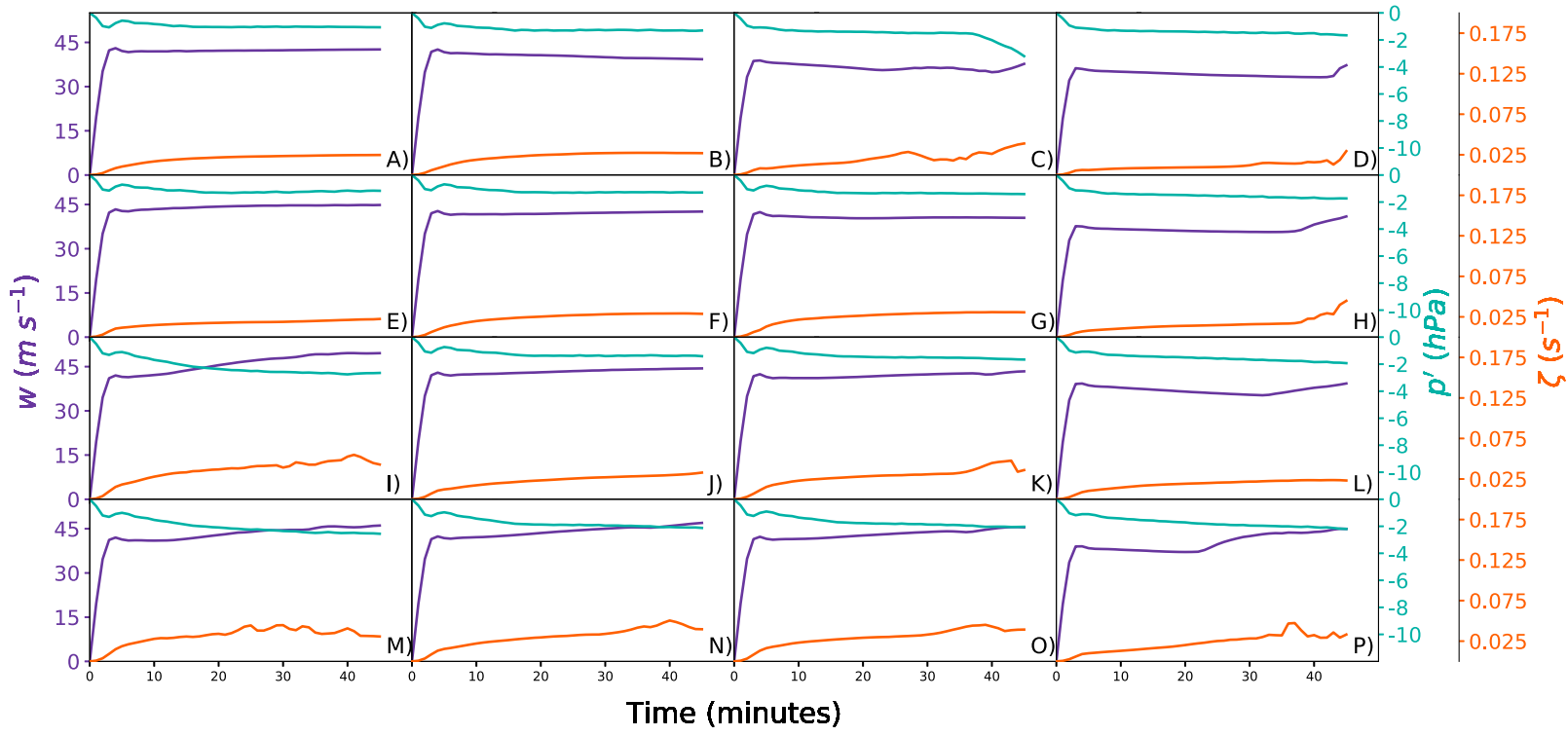

FIG. 9. As in Fig. 6, but for the 3-7 km AGL layer in each node of the GR suite (labeled by letters as in Fig. 2). 


\section{0 - $3.0 \mathrm{~km}$ AGL depth average}

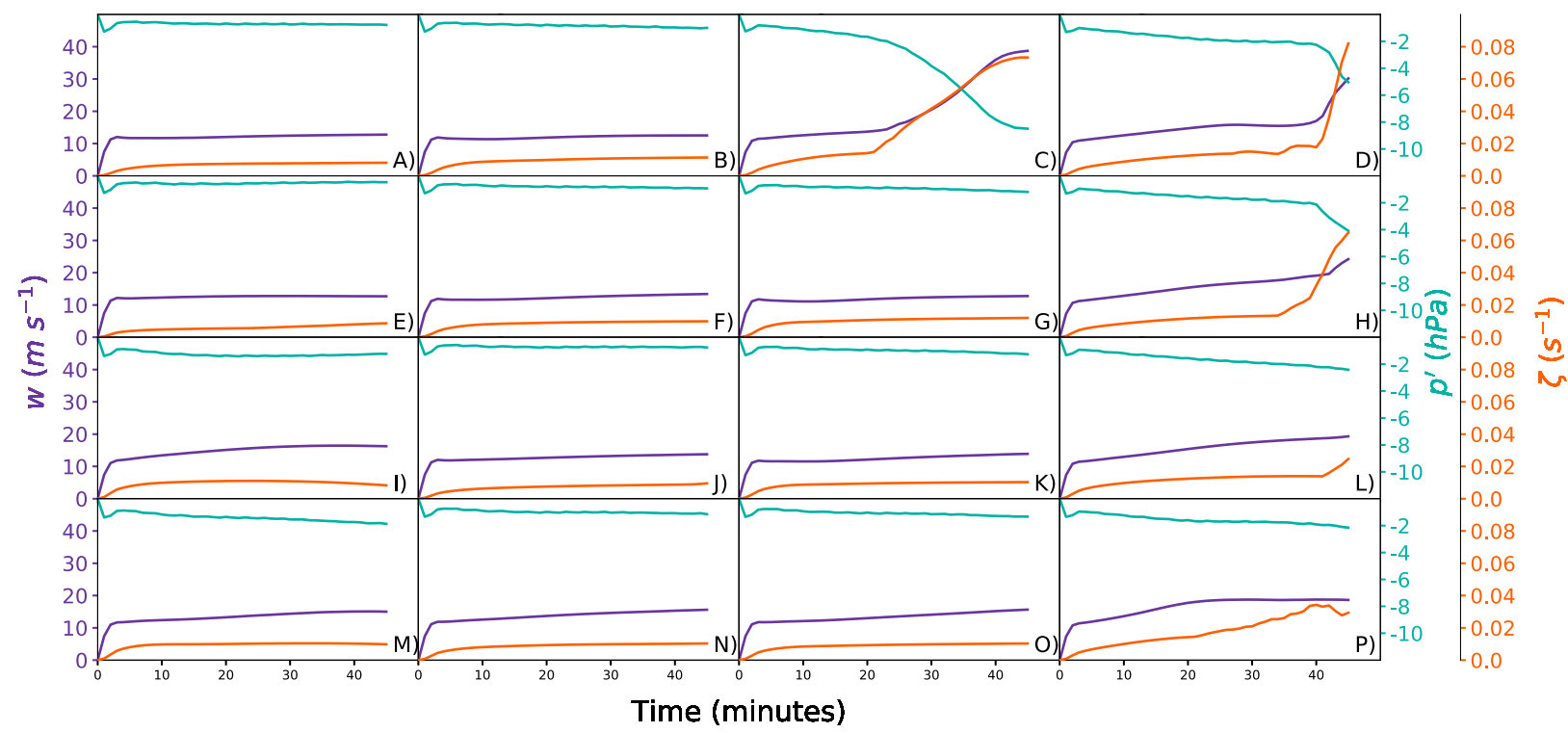

FIG. 10. As in Fig. 9, but for the 1-3 km AGL depth-averaged values.

environmental vertical wind profile, if any, must be almost entirely realized in the lower levels.

We next examine the layer in which a low-level mesocyclone typically occurs (1-3 km AGL). It is readily evident that emergent behaviors occur in a subset of the environments (Fig. 10). The majority of the profiles produce $1-3 \mathrm{~km} w$ and $\zeta$ that asymptotically reach $10-15 \mathrm{~m} \mathrm{~s}^{-1}$ and approximately $0.01 \mathrm{~s}^{-1}$, respectively. However, profiles C, D, and $\mathrm{H}$ exhibit a strong relationship among the dynamical fields which is consistent with the hypothesized dynamical response (Fig. 10). For profile C, around $20 \mathrm{~min}$ into the simulation, $\zeta$ and $p^{\prime}$ grow rapidly, with $w$ trailing shortly behind. When $p^{\prime}$ becomes steady after $40 \mathrm{~min}$, the $\zeta$ and $w$ fields also become steady with final values approaching $-8 \mathrm{hPa}, 0.075 \mathrm{~s}^{-1}$, and $40 \mathrm{~m} \mathrm{~s}^{-1}$, respectively. For profiles $\mathrm{D}$ and $\mathrm{H}$, the onset of this response is delayed, but toward the end of the 45-min run, both environments exhibit rapid growth with final amplitudes rivaling those in environment $\mathrm{C}$. Environments $\mathrm{L}$ and $\mathrm{P}$ show a hint of this response, but it is even more delayed and weaker.

We next ask to what extent these $1-3 \mathrm{~km}$ differences drive responses in the lowest $500 \mathrm{~m} \mathrm{AGL}$, where they would be most relevant to tornadogenesis. Since $500 \mathrm{~m}$ AGL is the base of $\dot{w}$ (i.e., where it goes to zero), $w$ enhancement at this level is solely from the dynamical response and not from our artificial updraft forcing. Most of the environments produce virtually no response in the three analyzed fields (Fig. 11), having values that are nearly indistinguishable from the quiescent control run (Fig. 6). In contrast, the same profiles that exhibit a dynamical response between 1 and $3 \mathrm{~km} \mathrm{AGL}(\mathrm{C}, \mathrm{D}, \mathrm{H}$, and $\mathrm{L}$ ) produce comparatively even stronger dynamical responses at $500 \mathrm{~m}$ AGL. In each case, the response increases $w$ by $>10 \mathrm{~m} \mathrm{~s}^{-1}$, increases $\zeta$ by $>0.05 \mathrm{~s}^{-1}$, and decreases $p^{\prime}$ by $>1.5 \mathrm{hPa}$.

The $0-500 \mathrm{~m} \mathrm{SRH}$ is closely related to the strength of the dynamical response displayed in Figs. 10 and 11. The CM1 environments with appreciable response all have 0-500 $\mathrm{m} \mathrm{SRH}$ values above the population median ( of $104 \mathrm{~m}^{2} \mathrm{~s}^{-2}$ ): profiles $\mathrm{C}$ $\left(162 \mathrm{~m}^{2} \mathrm{~s}^{-2}\right), \mathrm{D}\left(197 \mathrm{~m}^{2} \mathrm{~s}^{-2}\right), \mathrm{H}\left(151 \mathrm{~m}^{2} \mathrm{~s}^{-2}\right), \mathrm{L}\left(136 \mathrm{~m}^{2} \mathrm{~s}^{-2}\right)$, and $\mathrm{P}\left(112 \mathrm{~m}^{2} \mathrm{~s}^{-2}\right)$. However, several environments with similar $0-500 \mathrm{~m}$ SRH values, profiles B $\left(145 \mathrm{~m}^{2} \mathrm{~s}^{-2}\right), \mathrm{G}$ $\left(164 \mathrm{~m}^{2} \mathrm{~s}^{-2}\right)$, and $\mathrm{K}\left(114 \mathrm{~m}^{2} \mathrm{~s}^{-2}\right)$, yield no appreciable dynamical response (Fig. 10). We return to this finding later (in section 3e). Environment D also exhibits a temporary pause in pressure falls around $35 \mathrm{~min}$ (with corresponding decreases in $\zeta$ and $w$ ) before the fields resume their original trends. It is possible, as explained by Coffer et al. (2017), that the unsteadiness of this particular mesocyclone's growth could be attributable to the presence of comparatively large 0-500 $\mathrm{m} \omega_{\mathrm{c}}$ $\left(0.0109 \mathrm{~s}^{-1}\right.$; Table 2$)$. In fact, profiles $\mathrm{D}, \mathrm{H}, \mathrm{L}$, and $\mathrm{P}$ all have low-level $\omega_{\mathrm{c}}$ values much larger than that of profile $\mathrm{C}(\mathrm{C}$ : $0.0006 \mathrm{~s}^{-1}$; H: $0.0124 \mathrm{~s}^{-1}$; L: $0.0129 \mathrm{~s}^{-1}$; and P: $0.0164 \mathrm{~s}^{-1}$; Table 2). Perhaps for this reason, environment $\mathrm{C}$ exhibits the earliest dynamical response (around $20 \mathrm{~min}$ ) while D, H, L, and $\mathrm{P}$ take over $35 \mathrm{~min}$ to establish a strong response. In other words, even if an environment has sufficient $\omega_{\mathrm{s}}$ (and low-level $\mathrm{SRH}$ ) to promote the formation of a robust mesocyclone, the onset of the intensifying low-level lifting could still be delayed due to the presence of substantial $\omega_{\mathrm{c}}$. It is possible that such delays could cause some nearly tornadic storms to fail in producing timely stretching of surface $\zeta$ into a tornado.

These differences among the simulations suggest an important linkage between the wind profile and what appears to be a dynamical response. For more direct attribution of cause and effect, we next look at a decomposition of $p^{\prime}$ to evaluate relative contributions to the vertical accelerations in these simulations.

\section{d. Analysis of low-level pressure perturbations and accelerations}

As revealed in Fig. 12, for the majority of simulations (which produce no response), the pressure field is similar: steady in 


\section{$0.5 \mathrm{~km} \mathrm{AGL}$}

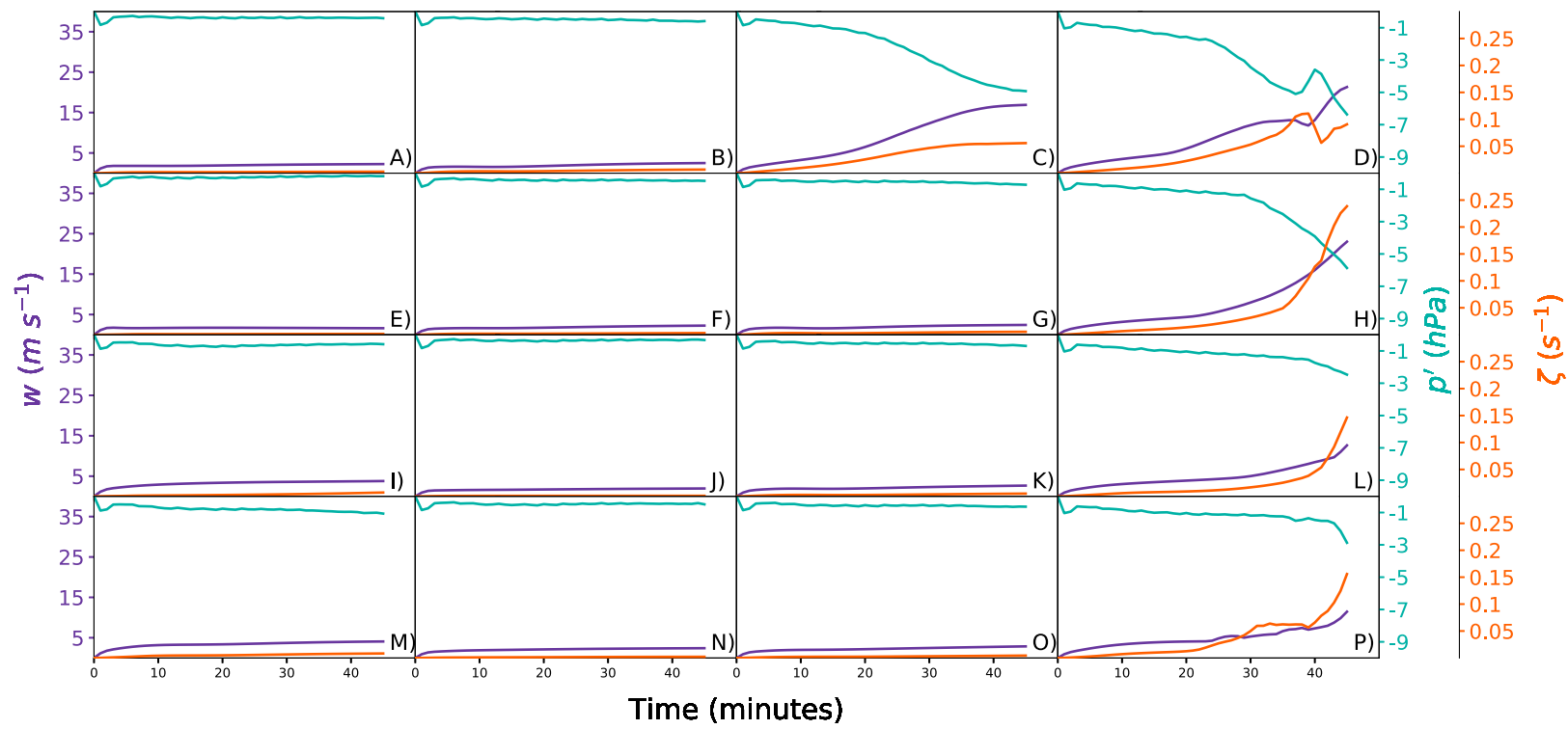

FIG. 11. As in Fig. 9, but for the $0.5 \mathrm{~km}$ AGL values.

time and rather undisturbed. There are noticeable buoyant pressure deficits $(0.5-1.0 \mathrm{hPa})$, which may seem surprising. Within our $0-12 \mathrm{~km}$ layer with adiabatic lapse rates, nonzero buoyancy results only from $p^{\prime}$ (since there is no moisture or thermal forcing). This effect has been demonstrated in tornado-like vortices with substantial $p_{\mathrm{D}}^{\prime}$ minima by Dawson et al. (2016). Here, the developing updrafts produce weaker but nonnegligible $p_{\mathrm{D}}^{\prime}$ minima associated with their mesocyclones combined with the updraft-in-shear effect (e.g., Fig. 8). As shown by Dawson et al. (2016), this lowered pressure is associated with lowered density (and thus positive buoyancy). Beneath this zone of positive buoyancy is lowered $p_{\mathrm{B}}^{\prime}$, which is

\section{$0.5 \mathrm{~km}$ AGL pressure perturbations}

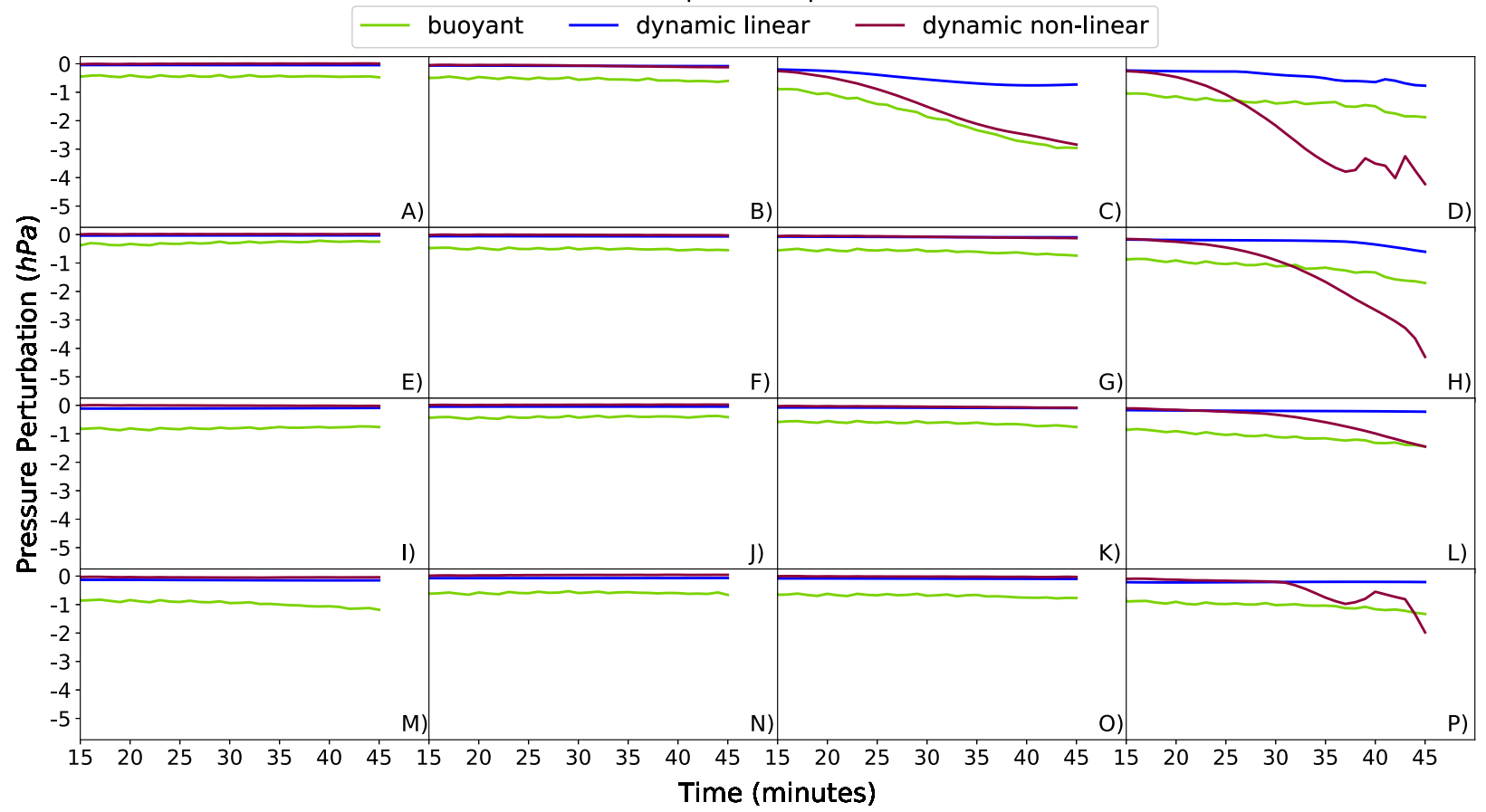

FIG. 12. A decomposition of the pressure perturbation field $\left(p^{\prime}, \mathrm{hPa}\right)$ at $0.5 \mathrm{~km}$ AGL into the contributions from buoyancy $\left(p_{\mathrm{B}}^{\prime}\right.$, yellowgreen), dynamic linear $\left(p_{\mathrm{DL}}^{\prime}\right.$, blue $)$, and dynamic nonlinear $\left(p_{\mathrm{DNL}}^{\prime}\right.$, red-violet) components during the final 30 min of the simulations. 


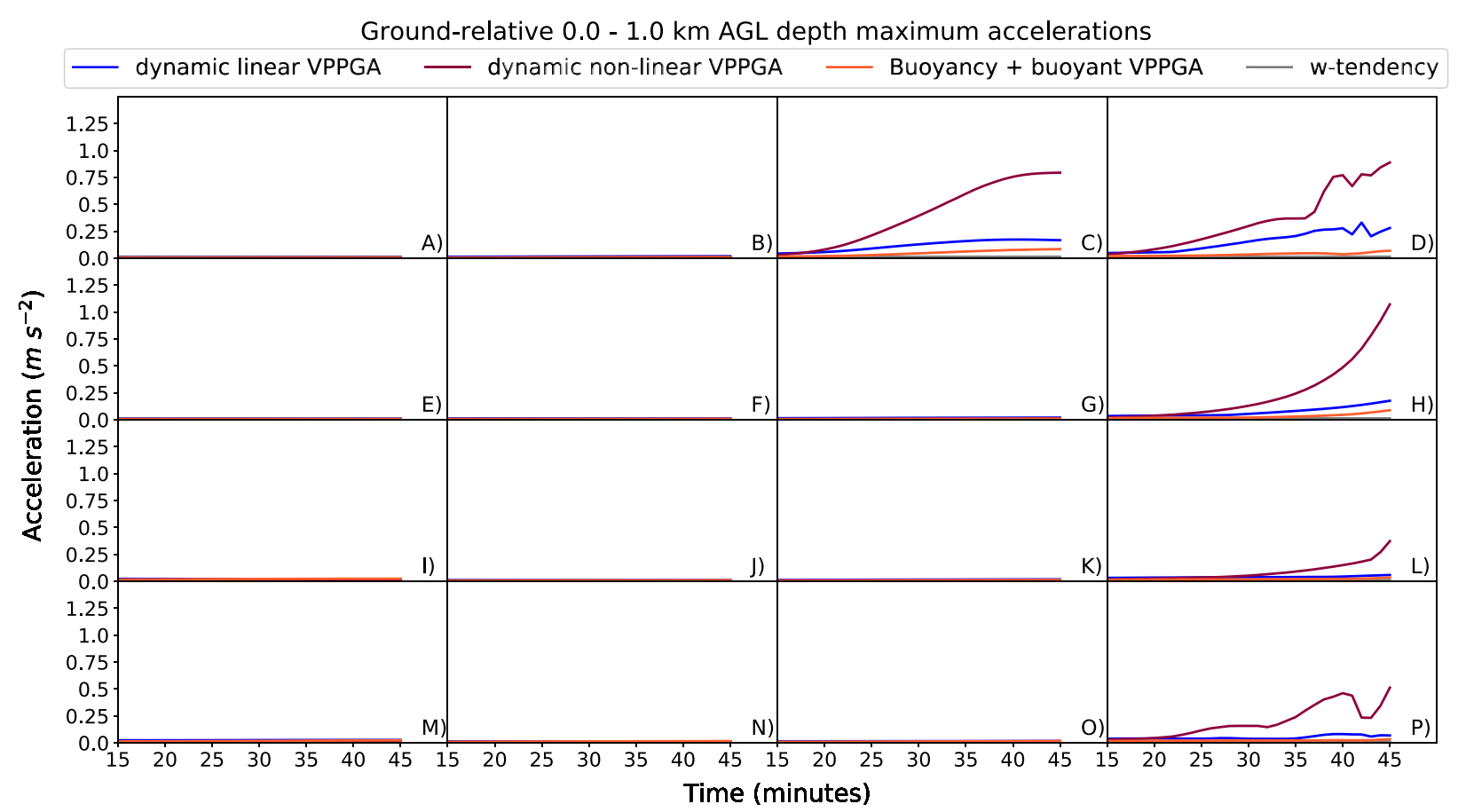

FIG. 13. Computational time series of $0-1 \mathrm{~km}$ AGL maximum vertical perturbation pressure gradient accelerations with dynamic linear (VPPGADL; blue) and dynamic nonlinear (VPPGADNL; red-violet) contributions $\left(\mathrm{m} \mathrm{s}^{-2}\right)$. Buoyancy $(B)$ and the buoyant vertical perturbation pressure gradient acceleration contribution (VPPGAB) are included as a summation $\left(B+\mathrm{VPPGAB}, \mathrm{m} \mathrm{s}^{-2}\right.$, red-orange). The $0-1 \mathrm{~km}$ AGL maximum $w$-tendency value $\left(\dot{w}, 0.013 \mathrm{~m} \mathrm{~s}^{-2}\right.$, gray) is included for reference. The period shown is for the final $30 \mathrm{~min}$ of each simulation in the ground-relative suite.

what appears in Fig. 12. The resultant vertical gradient in $p_{\mathrm{B}}^{\prime}$ nearly offsets the buoyancy to yield near-zero ACCB (i.e., $B+$ VPPGAB; Fig. 13).

The simulations with a strong low-level dynamical response $(\mathrm{C}, \mathrm{D}$, and $\mathrm{H})$ also have a small contribution from $p_{\mathrm{DL}}^{\prime}$ (Fig. 12). This is due to the updraft-in-shear effect and emerges in most supercellular environments (which have strong vertical wind shear). But, the dynamic nonlinear component (associated with local rotation) is the most distinctive contributor to the simulated pressure declines in C, D, and H (Fig. 12). The same evolution occurs in environments $\mathrm{L}$ and $\mathrm{P}$ but to a lesser degree, which is consistent with their more modest dynamical responses as identified in section $3 \mathrm{c}$. The remaining simulations have a negligible $p_{\mathrm{D}}^{\prime}$, signaling the absence of a rotationally induced response in these environments. In short, large $p_{\mathrm{D}}^{\prime}$ falls are unique to the runs producing low-level updraft intensification. Due to the importance of low-level vertical accelerations in tornadogenesis, we next analyze the associated dynamic VPPGA in the lowest $1 \mathrm{~km}$ (i.e., centered on $500 \mathrm{~m}$ AGL; Fig. 13).

As discussed above, the net buoyant accelerations are small (by experimental design; Fig. 13). The $0-1 \mathrm{~km}$ maximum value from our artificial $\dot{w}\left(0.013 \mathrm{~m} \mathrm{~s}^{-2}\right)$ is included for reference but is also negligible (Fig. 13) since its base is at $500 \mathrm{~m}$ AGL in our simulations. Environments C, D, H, L, and P produce differing growth rates and partitioning among components, but all show that the dynamic nonlinear VPPGA (VPPGADNL) is the main contributor to the enhancement in $w$ (Fig. 13). Since the low-level dynamical response is a rotationally driven process, VPPGADNL can be said to quantify the low-level dynamical response. The magnitude of this effect easily outweighs all other contributors in $\mathrm{C}, \mathrm{D}, \mathrm{H}, \mathrm{L}$, and $\mathrm{P}$, but it is completely absent in the other simulations.

It has long been known that the dynamic acceleration components in a supercell's updraft can exceed the buoyant acceleration components (e.g., McCaul et al. 1996; Weisman and Rotunno 2000; Coffer and Parker 2015). One of our stated goals was to quantify the magnitude of the purported supercell dynamical response and its importance relative to buoyancy. In the simulations with the greatest response, VPPGADNL exceeded $1.0 \mathrm{~m} \mathrm{~s}^{-2}$ (Fig. 13). Such values would far exceed the typical buoyant accelerations found in real supercells (commonly peaking in midlevels at $0.1-0.3 \mathrm{~m} \mathrm{~s}^{-2}$; as in Peters et al. 2019). Similar accelerations are observed in environments $C$, $\mathrm{D}, \mathrm{H}$, and L corresponding to the SRR suite of environments $\left(0.80 \mathrm{~m} \mathrm{~s}^{-2}\right.$, Fig. 14). Again, these accelerations are almost entirely due to VPPGADNL, and these nodes correspond to the highest 0-500 $\mathrm{m}$ SRH values in the SRR suite (Fig. 3).

The present simulations are conducted in a dry adiabatic troposphere, and so ascending air encounters no resistance from the ambient static stability (i.e., tropospheric temperature inversions and sub-dry-adiabatic environmental lapse rates). Thus, our VPPGADNL values may produce stronger updrafts than what storms experience in nature. On the other hand, in some high CAPE or weakly capped scenarios, the low-level buoyant accelerations may be larger than our artificial $\dot{w}$, which 
Storm-relative rotated $0.0-1.0 \mathrm{~km}$ AGL depth maximum accelerations

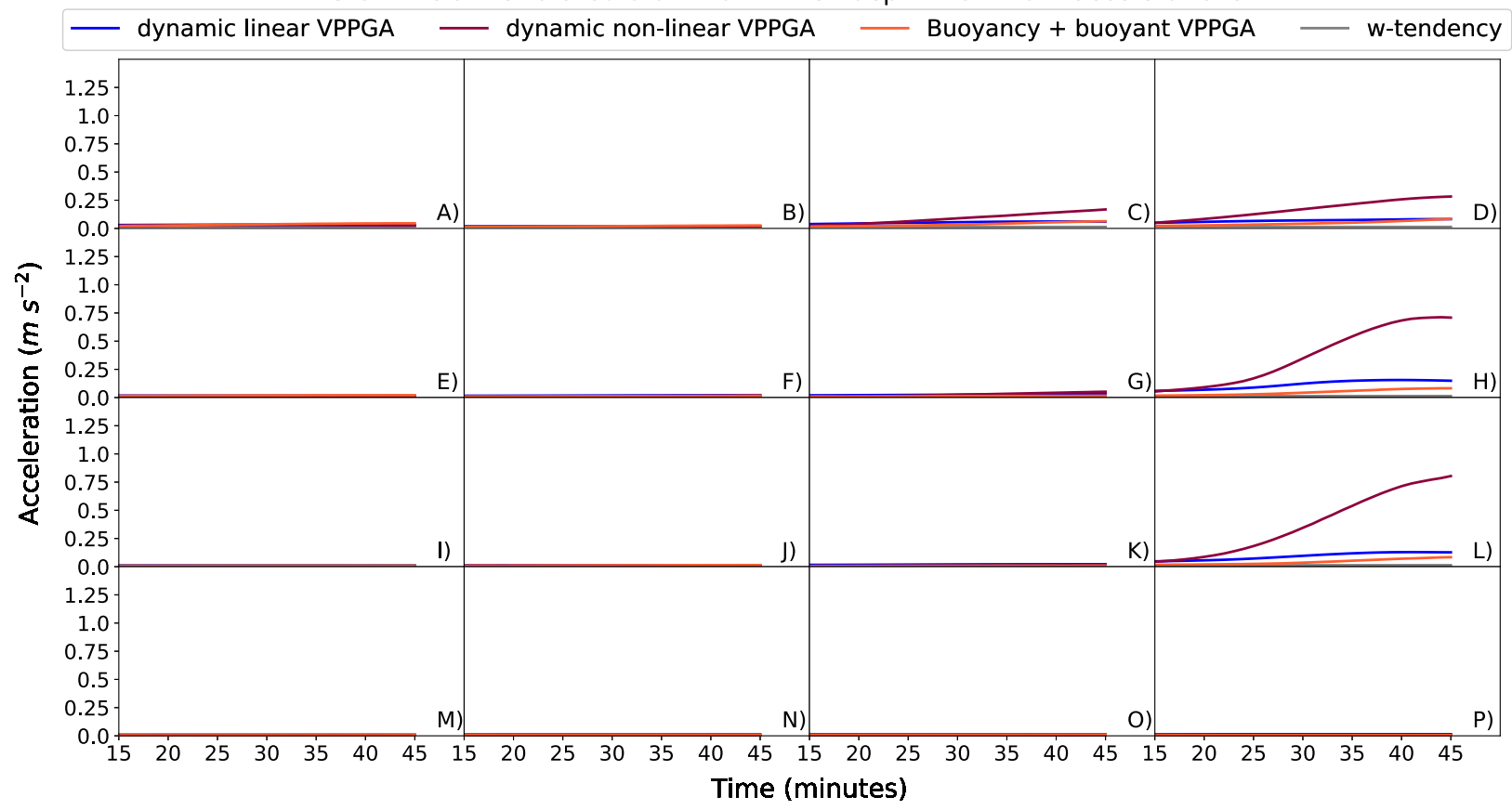

FIG. 14. As in Fig. 13, but for the storm-relative rotated suite.

could promote a stronger response. For reference, in an environment of $279 \mathrm{~m}^{2} \mathrm{~s}^{-2}$ of $0-1 \mathrm{~km} \mathrm{SRH}$ and $2600 \mathrm{~J} \mathrm{~kg}^{-1}$ of CAPE, Coffer and Parker (2015) simulated a full physics supercell with $0-1 \mathrm{~km}$ AGL maximum total dynamic VPPGA near $0.40 \mathrm{~m} \mathrm{~s}^{-2}$, which exceeded the maximum buoyant accelerations by roughly a factor of 4 .

It is the relationship of large dynamic lifting to large lowlevel SRH that emerges as most important from this analysis. Most right-moving supercell environments do not produce this dynamical enhancement (Figs. 11-14); only the profiles with the highest SRH achieve it. Given the importance of low-level stretching to tornadogenesis, it is unsurprising that these are also the environments in the SOMs with the highest \%ST. Because the near-ground updraft enhancements are driven primarily by VPPGADNL, they could conceivably overcome appreciable negative buoyancy such as is found in the vorticityrich outflow of real storms. To shed more light on the nature of the VPPGADNL within these storms, we look at vertical $(x-z)$ cross sections through the centerlines of the resultant updrafts (Figs. 15 and 16).

In our GR environment $\mathrm{C}$, which has a strong, steady lowlevel response (e.g., Fig. 11), the VPPGADNL is shown to be contained entirely below $1.5 \mathrm{~km}$ AGL (Fig. 15), with substantial upward accelerations toward a pressure minimum at that level followed by negative accelerations above it. The other GR cases with the most robust low-level dynamical responses (environments D and $\mathrm{H}$ ) have a similar structure (Fig. 15) but with VPPGADNL that is concentrated even closer to the ground due to the lower altitude of the pressure minimum (between 0.5 and $1 \mathrm{~km}$ AGL). The simulations with a response in the SRR suite tell a similar tale (Fig. 16). There is an interesting trend along the SRR suite's top row (Figs. 16a-d) wherein the VPPGADNL is stronger and resides lower in the troposphere with increasing low-level SRH and $\omega_{\mathrm{s}}$. A related impact is that the updrafts themselves exhibit "base lowering" (i.e., increased values of $w$ at lower levels) for environments with strong low-level vertical wind shear (Figs. 15, 16). It is also potentially relevant that the maximum in VPPGADNL is in some environments seen to occur above $1 \mathrm{~km}$ AGL (e.g., Fig. 16, simulations A-D). The SOM's \%ST is comparatively diminished in such environments, perhaps because in nature these accelerations would be too far aloft to assist in lifting and stretching vorticityrich, near-surface circulations.

Interestingly, a subset of recent literature has attempted to link updraft width to supercells' intensity (e.g., Trapp et al. 2017, 2018; Warren et al. 2017; Peters et al. 2019; Sessa and Trapp 2020), with some implications that this may determine tornadogenesis. Though our investigation was not motivated by the role of updraft width in the intensification of the lowlevel updraft and rotation, the results of our study may shed light on this active research area. Because the imposed updraft forcing is held fixed among runs, we find very little change in updraft width among simulations (Figs. 15, 16). We also find very little difference in the peak updraft velocities found in midlevels (Figs. 9, 15, 16). Rather, across a range of environments, the dynamical intensification process that seems most relevant to tornadogenesis is entirely manifested in the lowest $\sim 1 \mathrm{~km}$ AGL. It appears that the near-ground wind profile modulates the production of this response, independent of updrafts' widths or midlevel velocities. We thus conclude our results in the next section by directly comparing a range of 

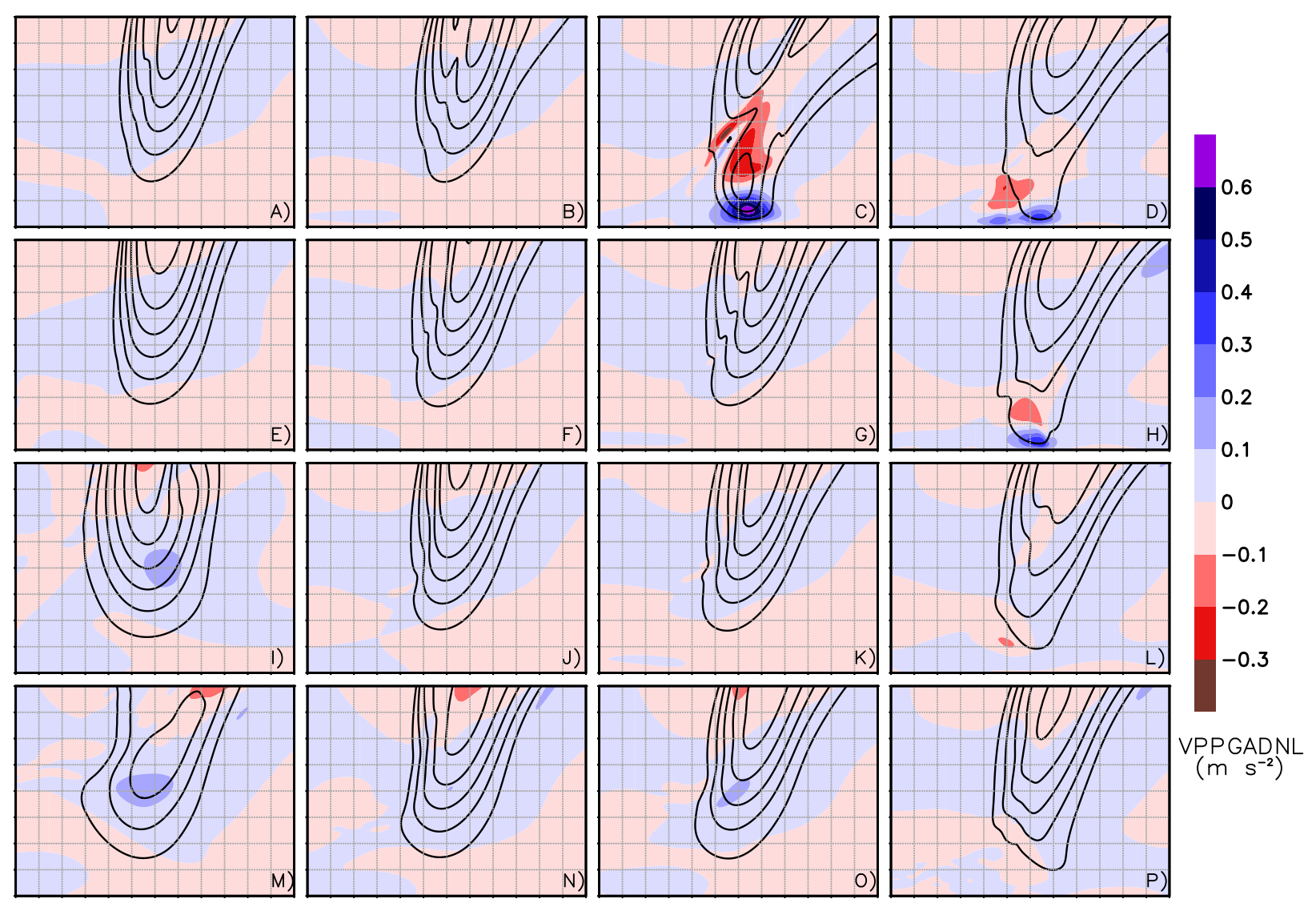

FIG. 15. Vertical $(x-z)$ cross sections through the approximate center of the updraft time averaged over the final $10 \mathrm{~min}$ of each simulation in the ground-relative (GR) suite. Vertical velocity $(w)$ is contoured in black (every $10 \mathrm{~m} \mathrm{~s}^{-1}$ ), and the dynamic nonlinear vertical perturbation pressure gradient acceleration (VPPGADNL) is shaded as shown (in $\mathrm{m} \mathrm{s}^{-2}$ ). Because this figure displays timeaveraged composites over $10 \mathrm{~min}$, there is some departure from the instantaneous values shown in prior figures. Height AGL (km) is displayed on the ordinate and $x(\mathrm{~km})$ is displayed on the abscissa. Each grid square is representative of $1 \mathrm{~km}^{2}$.

environmental parameters $\left(\mathrm{SRH}, \omega_{\mathrm{s}}\right.$, and $\left.\omega_{\mathrm{c}}\right)$ to the simulated updraft dynamics.

\section{e. Modeling results in the context of SOM statistics}

Environments exhibiting a low-level dynamical response in the model are exclusively those with at least $110 \mathrm{~m}^{2} \mathrm{~s}^{-2}$ of 0 $500 \mathrm{~m} \mathrm{SRH}$ (Fig. 17); these environments have associated 0$500 \mathrm{~m} \omega_{\mathrm{s}}$ in excess of $0.015 \mathrm{~s}^{-1}$ (e.g., Fig. 4). In the cases producing a clear response, the upward-directed VPPGADNL ranges from 0.17 to $1.07 \mathrm{~m} \mathrm{~s}^{-2}$. In turn, for these environments, much higher significant tornado probabilities occur in nature (shading of data points in Fig. 17). An important finding is that most right-moving supercell environments do not achieve such low-level dynamical responses. It seems clear that prolific ST environments are "special" in that they support a truly distinctive dynamical enhancement of low-level $w$ (and thus $\zeta$ stretching which could accomplish tornadogenesis).

We earlier mentioned that at least some of the higher SRH environments did not produce a response; these environments also tended to have lower \% ST in the SOMs (shading of data points in Fig. 17). Coffer and Parker (2017), Coffer et al. (2017), Coffer and Parker (2018), and Murdzek et al. (2020) have suggested that appreciable $\omega_{\mathrm{c}}$ may lead to interruption of the low-level mesocyclone which could hinder subsequent tornadogenesis. This may be true in some cases (as described in section 3c), but Fig. 18 shows that strong dynamical responses occur across a wide range of $\omega_{\mathrm{c}}$ values provided that $0-500 \mathrm{~m}$ SRH is above a certain threshold. The $0-500 \mathrm{~m}$ ratio of $\omega_{\mathrm{s}}$ to $\omega_{\mathrm{c}}$ (Fig. S9 in the online supplemental materials) is also poorly related to the VPPGADNL values; overall, many supercells of varying types occur in environments with $0-500 \mathrm{~m} \omega_{\mathrm{s}}: \omega_{\mathrm{c}}$ ratios between 2 and 4 . The varying sensitivity to $\omega_{\mathrm{c}}$ may mean that its effects are dependent on other parameters (e.g., SRF). Further analysis of such effects would be of interest in the future.

By design, our experiment has specifically isolated the effects of the environmental wind profile upon a controlled updraft. All of the environments represented by our SOMs were associated with severe supercells. Notably, at least some observed tornadoes (including significant ones) are present in the SOM nodes that do not produce a response in our simulations. This suggests that although the low-level wind profile is extremely important, it is not the sole ingredient that determines whether a supercell will be tornadic. Other environmental ingredients and within storm processes must play a role. For 

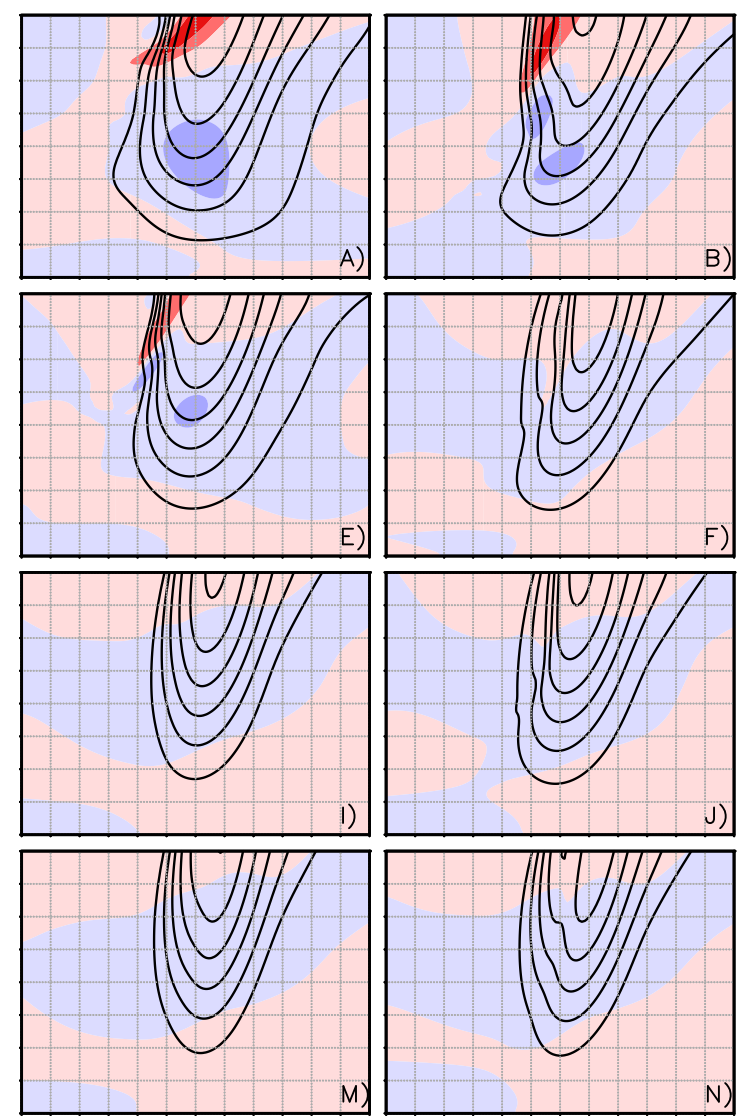
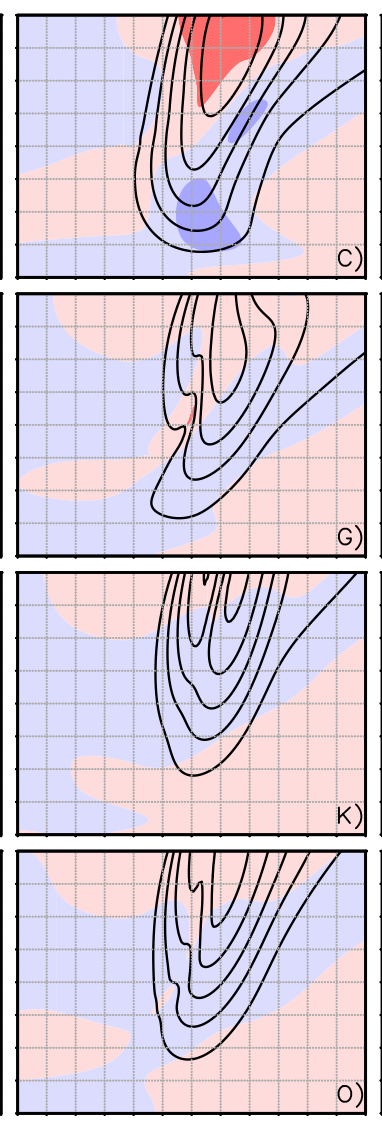

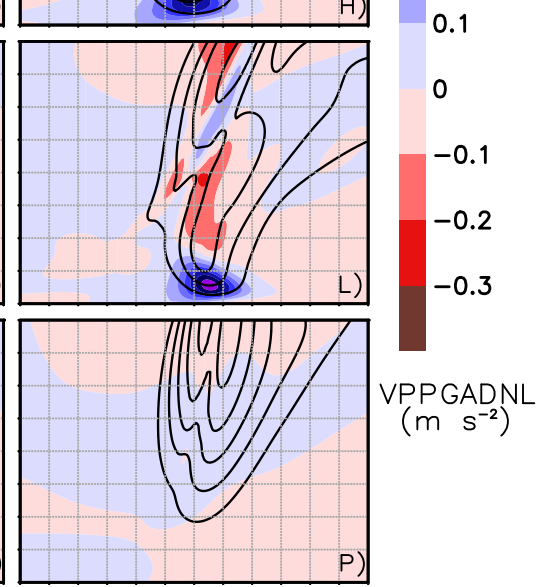

FIG. 16. As in Fig. 15, but for the storm-relative rotated (SRR) suite.

example, Fischer and Dahl (2020) showed that both the lowlevel updraft and the surface cold pool characteristics modulate the tornadogenesis process. And, to some degree, the process in real storms is likely stochastic (e.g., Markowski 2020). However, by omitting thermodynamic variations and precipitation-driven processes from our experimental design, we have shown that the near-ground wind profile likely has major influence.

\section{Synthesis}

\section{a. Summary}

Forecasters and scientists have long sought to discriminate tornadic from nontornadic supercells. It has recently been shown that $0-500 \mathrm{~m} \mathrm{SRH}$ is both operationally and dynamically meaningful in identifying significantly tornadic $(\mathrm{EF} 2+)$ versus nontornadic supercells (Coffer et al. 2019). This linkage is hypothesized to occur via a supercell-induced dynamical response whereby updraft rotation yields an intensifying upward VPPGA (Coffer and Parker 2017, 2018; Coffer et al. 2017; Flournoy et al. 2020), which can lift near-surface, vorticity-rich air during tornadogenesis. Given these recent findings, we asked the following questions:

1) Is there an identifiable subset of wind profiles over which the link connecting the low-level environmental winds, low-level dynamical response, and tornadogenesis probabilities is most prominent?

2) How much does the low-level dynamical response contribute to enhancements in low-level vertical velocity $(w)$ and low-level vertical vorticity $(\zeta)$ ?

To address these problems, we utilized a large dataset of 20194 environmental wind profiles from tornadic and nontornadic events and identified recurring low-level wind profiles using machine learning (self-organizing maps). The resultant wind profiles were then used in a controlled, idealized numerical experiment. This enabled us to directly relate the updraft evolution to differences in the low-level wind profiles.

\section{b. Conclusions}

Based on the numerical experiments, our primary conclusions are as follows:

- With our controlled updraft forcing, the wind profile itself produces very little variation in midlevel (3-7 km AGL) updraft and mesocyclone structure (intensity and width) across environments that foster right-moving supercells.

- Environments that do not foster a dynamical response have low-level updrafts with quasi-steady properties, including minimal vertical velocities at or below $500 \mathrm{~m}$ AGL.

- When present, a low-level dynamical response can increase the vertical velocity $(w)$ by up to $25 \mathrm{~m} \mathrm{~s}^{-1}$, increase the vertical 


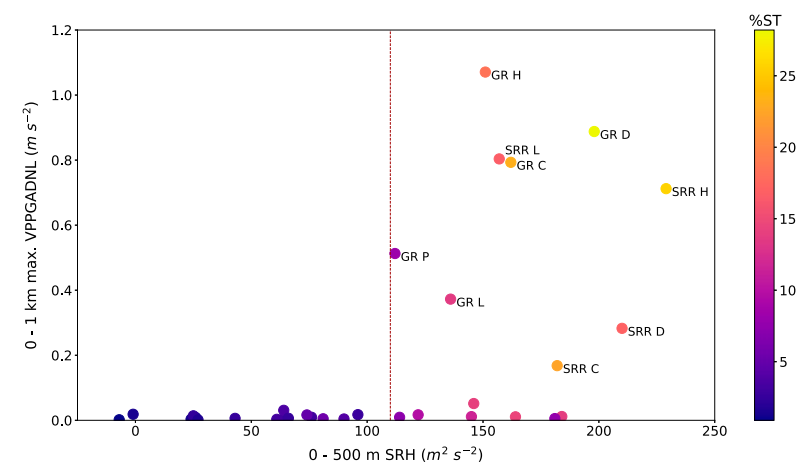

FIG. 17. A 3D scatterplot depicting 0-500 $\mathrm{m} \mathrm{SRH}\left(\mathrm{m}^{2} \mathrm{~s}^{-2}\right.$, abscissa), 0-1 km maximum dynamic nonlinear vertical perturbation pressure gradient acceleration (VPPGADNL; $\mathrm{m} \mathrm{s}^{-2}$, ordinate), and significant tornado percentage (\%ST, color) for the combined GR and SRR simulations. The $0-500 \mathrm{~m}$ SRH values are computed using the final domain translation motions. The dark red dashed line denotes $110 \mathrm{~m}^{2} \mathrm{~s}^{-2}$. Environments with a prevalent dynamical response have been labeled for the ground-relative "GR" and storm-relative rotated "SRR" frameworks.

vorticity ( $\zeta$ ) by up to $0.2 \mathrm{~s}^{-1}$, and decrease the pressure perturbation $\left(p^{\prime}\right)$ by up to $5 \mathrm{hPa}$ at a height of $500 \mathrm{~m} \mathrm{AGL}$ (i.e., below where any artificial updraft forcing was present). Such near-ground enhancement would presumably be directly relevant to the stretching of near-ground vertical vorticity required to accomplish tornadogenesis.

- Low-level dynamical responses emerge in environments with large low-level (0-500 m AGL) environmental stormrelative helicity $\left(\mathrm{SRH}>110 \mathrm{~m}^{2} \mathrm{~s}^{-2}\right)$ and streamwise vorticity $\left(\omega_{\mathrm{s}}>0.015 \mathrm{~s}^{-1}\right)$. However, not all environments that fit these criteria produce the dynamical response. The lowlevel wind profile may be an important but not sole predictor of tornadogenesis, and other within storm processes and environmental ingredients also must play a role.

- This dynamical response occurs across a range of environmental $0-500 \mathrm{~m}$ crosswise vorticity $\left(\omega_{\mathrm{c}}\right)$ values within this parameter space. This supports Coffer et al.'s (2019) conclusion that $\omega_{\mathrm{c}}$ is probably not an effective discriminator between significantly tornadic and nontornadic right-moving supercells. We found no relationship between $0-500 \mathrm{~m} \omega_{\mathrm{c}}$ and significant tornado percentages in the self-organizing maps. However, environmental $\omega_{\mathrm{c}}$ could still be responsible for delaying the onset of the dynamical response.

- The enhancement of the low-level updraft is via VPPGADNL. Our simulations produce acceleration values up to 10 times larger than the buoyancy-driven accelerations from supercells that have been previously simulated in realistic full-physics environments.

The self-organizing maps trained on $0-500 \mathrm{~m}$ AGL $(u, v)$ wind profiles reveal strong linkages between environments with large near-ground SRH, appreciable modeled updraft intensification, and observed significant tornado percentages. Our results show that intense dynamic lifting of near-surface air (which appears to be the "make-or-break" moment of tornadogenesis) is most likely to occur in environments where

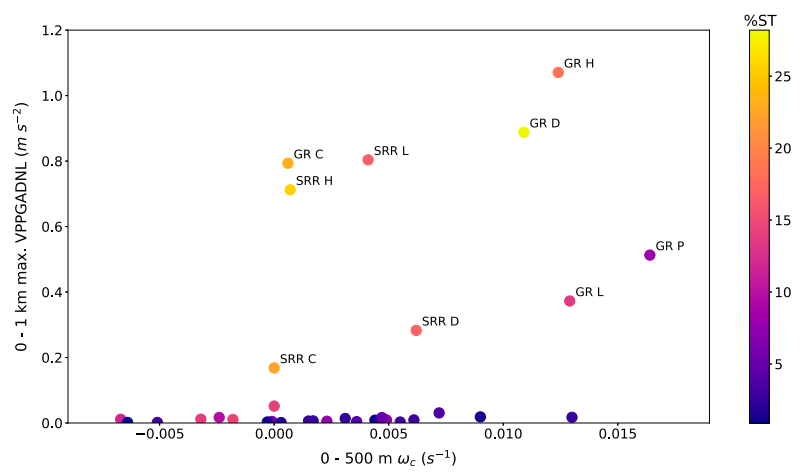

FIG. 18. As in Fig. 17, but with $0-500 \mathrm{~m}$ crosswise vorticity $\left(\omega_{\mathrm{c}}, \mathrm{s}^{-1}\right)$ on the abscissa. The $0-500 \mathrm{~m}$ crosswise vorticity values are computed using the final domain translation motions. Environments with a prevalent dynamical response have been labeled for the groundrelative "GR" and storm-relative rotated "SRR" frameworks.

0-500 $\mathrm{m}$ SRH exceeds some threshold (in this experimental design, $110 \mathrm{~m}^{2} \mathrm{~s}^{-2}$ ). When it occurs, this dynamical response appears to far outpace any effects of low-level buoyancy. A subsequent feedback owing to this response could conceivably ensue if this enhanced updraft leads to added tilting and stretching of the environmental vorticity (further enhancing updraft rotation, $p_{\mathrm{DNL}}^{\prime}$, and so on). Such a nonlinear process could dramatically strengthen updrafts in the subset of environments where it occurs.

\section{c. Future work}

The conclusions we have presented in turn prompt new questions. What processes occur in nature that lead to occasional significant tornadoes in lower SRH environments and tornadogenesis failure in higher SRH environments? Do tornadoes ever form without this low-level dynamical response? If so, by what other process is the final (step 3) stretching accomplished? If not, then what processes preclude or promote the formation of a low-level dynamical response in the environments with moderate $0-500 \mathrm{~m} \mathrm{SRH} \mathrm{(e.g.,} \approx 110-185 \mathrm{~m}^{2} \mathrm{~s}^{-2}$ ) and $\omega_{\text {s }}$ (e.g., $\approx 0.015-0.027 \mathrm{~s}^{-1}$ ) wherein only some environments produced a response? These questions likely require work using full-physics simulations wherein thermodynamic and microphysical processes within supercells can produce a wider range of outcomes. We view the present study as a first attempt to quantify one of the main building blocks of the tornadogenesis process that should be evident even in extremely complex storm observations and simulations.

Acknowledgments. This project was supported by NSF Grant AGS-1748715. We thank Dr. George Bryan for his enduring maintenance of CM1 and to NCAR for their computing services (Cheyenne, doi:10.5065/D6RX99HX). The authors would also like to recognize Drs. Sandra Yuter and Gary Lackmann for their recommendations to improve an earlier version of this study and manuscript as well as the members of the Convective Storms Group at North Carolina State University (Dr. Andrew Wade, Dr. Brice Coffer, Levi Lovell, and Adam Werkema) for their assistance with and suggestions 
for model configuration and graphical display techniques. The authors also thank John Hart, Ryan Jewell, Bryan Smith, and Rich Thompson of the NOAA/NWS/NCEP Storm Prediction Center for their efforts in developing the RUC/RAP SFCOA proximity sounding database, designating the convective mode of each storm report, and sharing the data with the community. We appreciate the editorial work performed by Dr. Louis Wicker and the feedback from three anonymous reviewers who enhanced the quality of this manuscript.

Data availability statement. All input soundings and model configuration files for the simulations are available from https://doi.org/10.5061/dryad.z8w9ghx9t. The code used for the self-organizing maps is available from https://doi.org/10.5281/ zenodo.4645813. Other large output datasets are available by request from the authors.

\section{REFERENCES}

Adlerman, E. J., K. K. Droegemeier, and R. Davies-Jones, 1999: A numerical simulation of cyclic mesocyclogenesis. J. Atmos. Sci., 56, 2045-2069, https://doi.org/10.1175/1520-0469(1999) $056<2045$ :ANSOCM $>2.0$.CO;2.

Anderson-Frey, A. K., Y. P. Richardson, A. R. Dean, R. L. Thompson, and B. T. Smith, 2016: Investigation of nearstorm environments for tornado events and warnings. Wea. Forecasting, 31, 1771-1790, https://doi.org/10.1175/WAF-D16-0046.1.

,,,,---- and,- 2017 : Self-organizing maps for the investigation of tornadic near-storm environments. Wea. Forecasting, 32, 1467-1475, https://doi.org/10.1175/WAF-D17-0034.1.

Benjamin, S. G., and Coauthors, 2004: An hourly assimilation-forecast cycle: The RUC. Mon. Wea. Rev., 132, 495-518, https://doi.org/ 10.1175/1520-0493(2004)132<0495:AHACTR > 2.0.CO;2.

—_, and Coauthors, 2016: A North American hourly assimilation and model forecast cycle: The Rapid Refresh. Mon. Wea. Rev., 144, 1669-1694, https://doi.org/10.1175/MWR-D-15-0242.1.

Blanchard, D. O., and J. M. Straka, 1998: Some possible mechanisms for tornadogenesis failure in a supercell. Preprints, 19 th Conf. on Severe Local Storms, Minneapolis, MN, Amer. Meteor. Soc., 116-119.

Bothwell, P. J., J. Hart, and R. L. Thompson, 2002: An integrated three-dimensional objective analysis scheme in use at the Storm Prediction Center. 21st Conf. on Severe Local Storms, San Antonio, TX, Amer. Meteor. Soc., JP3.1, https://ams.confex.com/ams/SLS_WAF_NWP/techprogram/ paper_47482.htm.

Brotzge, J., S. Erickson, and H. Brooks, 2011: A 5-yr climatology of tornado false alarms. Wea. Forecasting, 26, 534-544, https:// doi.org/10.1175/WAF-D-10-05004.1.

Bryan, G. H., and J. M. Fritsch, 2002: A benchmark simulation for moist nonhydrostatic numerical models. Mon. Wea. Rev., 130, 2917-2928, https://doi.org/10.1175/1520-0493(2002)130<2917: ABSFMN $>2.0 . \mathrm{CO} ; 2$.

Bunkers, M. J., 2018: Observations of right-moving supercell motion forecast errors. Wea. Forecasting, 33, 145-159, https:// doi.org/10.1175/WAF-D-17-0133.1.

—, B. A. Klimowski, J. W. Zeitler, R. L. Thompson, and M. L. Weisman, 2000: Predicting supercell motion using a new hodograph technique. Wea. Forecasting, 15, 61-79, https://doi.org/ 10.1175/1520-0434(2000)015<0061:PSMUAN>2.0.CO;2.
—, J. S. Johnson, L. J. Czepyha, J. M. Grzywacz, B. A. Klimowski, and M. R. Hjelmfelt, 2006: An observational examination of long-lived supercells. Part II: Environmental conditions and forecasting. Wea. Forecasting, 21, 689-714, https://doi.org/10.1175/WAF952.1.

Coffer, B. E., and M. D. Parker, 2015: Impacts of increasing lowlevel shear on supercells during the early evening transition. Mon. Wea. Rev., 143, 1945-1969, https://doi.org/ 10.1175/MWR-D-14-00328.1.

$\longrightarrow$, and - , 2017: Simulated supercells in nontornadic and tornadic VORTEX2 environments. Mon. Wea. Rev., 145, 149180, https://doi.org/10.1175/MWR-D-16-0226.1.

— , and — 2018: Is there a "tipping point" between simulated nontornadic and tornadic supercells in VORTEX2 environments? Mon. Wea. Rev., 146, 2667-2693, https://doi.org/ 10.1175/MWR-D-18-0050.1.

— — J. M. L. Dahl, L. J. Wicker, and A. J. Clark, 2017: Volatility of tornadogenesis: An ensemble of simulated nontornadic and tornadic supercells in VORTEX2 environments. Mon. Wea. Rev., 145, 4605-4625, https://doi.org/ 10.1175/MWR-D-17-0152.1.

- — - R. L. Thompson, B. T. Smith, and R. E. Jewell, 2019: Using near-ground storm relative helicity in supercell tornado forecasting. Wea. Forecasting, 34, 1417-1435, https://doi.org/ 10.1175/WAF-D-19-0115.1.

—, M. Taszarek, and M. D. Parker, 2020: Near-ground wind profiles of tornadic and nontornadic environments in the United States and Europe from ERA5 reanalyses. Wea. Forecasting, 35, 2621-2638, https://doi.org/10.1175/WAF-D20-0153.1.

Dahl, J. M. L., 2015: Near-ground rotation in simulated supercells: On the robustness of the baroclinic mechanism. Mon. Wea. Rev., 143, 4929-4942, https://doi.org/10.1175/MWR-D-15-0115.1.

Davenport, C. E., and M. D. Parker, 2015: Impact of environmental heterogeneity on the dynamics of a dissipating supercell thunderstorm. Mon. Wea. Rev., 143, 4244-4277, https://doi.org/10.1175/MWR-D-15-0072.1.

Davies-Jones, R., 1984: Streamwise vorticity: The origin of updraft rotation in supercell storms. J. Atmos. Sci., 41, 2991-3006, https:// doi.org/10.1175/1520-0469(1984)041<2991:SVTOOU > 2.0.CO;2. , 2015: A review of supercell and tornado dynamics. Atmos. Res., 158-159, 274-291, https://doi.org/10.1016/j.atmosres.2014.04.007.

— perspective. The Tornado: Its Structure, Dynamics, Prediction, and Hazards, Geophys. Monogr., Vol. 79, Amer. Geophys. Union, 105-114.

Dawson, I., T. Daniel, M. Xue, A. Shapiro, J. A. Milbrandt, and A. D. Schenkman, 2016: Sensitivity of real-data simulations of the 3 May 1999 Oklahoma City tornadic supercell and associated tornadoes to multimoment microphysics. Part II: Analysis of buoyancy and dynamic pressure forces in simulated tornado-like vortices. J. Atmos. Sci., 73, 1039-1061, https://doi.org/10.1175/JAS-D-15-0114.1.

Esterheld, J. M., and D. J. Giuliano, 2008: Discriminating between tornadic and non-tornadic supercells: A new hodograph technique. Electron. J. Severe Storms Meteor., 3 (2), https:// ejssm.org/ojs/index.php/ejssm/article/viewArticle/33.

Fischer, J., and J. M. L. Dahl, 2020: The relative importance of updraft and cold pool characteristics on supercell tornadogenesis in highly idealized simulations. J. Atmos. Sci., 77, 4089-4107, https://doi.org/10.1175/JAS-D-20-0126.1.

Flournoy, M. D., M. C. Coniglio, E. N. Rasmussen, J. C. Furtado, and B. E. Coffer, 2020: Modes of storm-scale variability and 
tornado potential in VORTEX2 near- and far-field tornadic environments. Mon. Wea. Rev., 148, 4185-4207, https:// doi.org/10.1175/MWR-D-20-0147.1.

Klees, A. M., Y. P. Richardson, P. M. Markowski, C. Weiss, J. M. Wurman, and K. K. Kosiba, 2016: Comparison of the tornadic and nontornadic supercells intercepted by VORTEX2 on 10 June 2010. Mon. Wea. Rev., 144, 3201-3231, https://doi.org/ 10.1175/MWR-D-15-0345.1.

Klemp, J. B., 1987: Dynamics of tornadic thunderstorms. Annu. Rev. Fluid Mech., 19, 369-402, https://doi.org/10.1146/ annurev.fl.19.010187.002101.

Kohonen, T., 1982: Self-organized formation of topologically correct feature maps. Biol. Cybern., 43, 59-69, https://doi.org/ 10.1007/BF00337288.

— , 1990: The self-organizing map. Proc. IEEE, 78, 1464-1480, https://doi.org/10.1109/5.58325.

_- 1997: Self-Organizing Maps. 2nd ed. Springer Series in Information Sciences, Springer-Verlag, 426 pp.

Liu, Y., R. H. Weisberg, and C. N. K. Mooers, 2006: Performance evaluation of the self-organizing map for feature extraction. J. Geophys. Res., 111, C05018, https://doi.org/10.1029/ $2005 \mathrm{JC} 003117$.

Markowski, P. M., 2016: An idealized numerical simulation investigation of the effects of surface drag on the development of near-surface vertical vorticity in supercell thunderstorms. J. Atmos. Sci., 73, 4349-4385, https://doi.org/10.1175/JAS-D16-0150.1.

- 2020: What is the intrinsic predictability of tornadic supercell thunderstorms? Mon. Wea. Rev., 148, 3157-3180, https:// doi.org/10.1175/MWR-D-20-0076.1.

— , and Y. P. Richardson, 2014: The influence of environmental low-level shear and cold pools on tornadogenesis: Insights from idealized simulations. J. Atmos. Sci., 71, 243-275, https:// doi.org/10.1175/JAS-D-13-0159.1.

_ J. M. Straka, and E. N. Rasmussen, 2002: Direct surface thermodynamic observations within the rear-flank downdrafts of nontornadic and tornadic supercells. Mon. Wea. Rev., 130, 1692-1721, https://doi.org/10.1175/1520-0493(2002)130<1692: DSTOWT $>2.0 . \mathrm{CO} ; 2$.

—, C. Hannon, J. Frame, E. Lancaster, A. Pietrycha, R. Edwards, and R. L. Thompson, 2003: Characteristics of vertical wind profiles near supercells obtained from the rapid update cycle. Wea. Forecasting, 18, 1262-1272, https://doi.org/10.1175/15200434(2003)018<1262:COVWPN>2.0.CO;2.

—, Y. Richardson, E. Rasmussen, J. Straka, R. Davies-Jones, and R. J. Trapp, 2008: Vortex lines within low-level mesocyclones obtained from pseudo-dual-Doppler radar observations. Mon. Wea. Rev., 136, 3513-3535, https://doi.org/10.1175/ 2008MWR2315.1.

__, _- M. Majcen, J. Marquis, and J. Wurman, 2011: Characteristics of the wind field in three nontornadic lowlevel mesocyclones observed by the Doppler on Wheels radars. Electron. J. Severe Storms Meteor., 6 (3), https:// www.ejssm.org/ojs/index.php/ejssm/article/viewArticle/75.

—_, and Coauthors, 2012: The pretornadic phase of the Goshen County, Wyoming, supercell of 5 June 2009 intercepted by VORTEX2. Part II: Intensification of low-level rotation. Mon. Wea. Rev., 140, 2916-2938, https://doi.org/10.1175/MWR-D11-00337.1.

McCaul, J., W. Eugene, and M. L. Weisman, 1996: Simulations of shallow supercell storms in landfalling hurricane environments. Mon. Wea. Rev., 124, 408-429, https://doi.org/10.1175/ 1520-0493(1996)124<0408:SOSSSI >2.0.CO;2.
Murdzek, S. S., P. M. Markowski, Y. P. Richardson, and R. L. Tanamachi, 2020: Processes preventing the development of a significant tornado in a Colorado supercell on 26 May 2010. Mon. Wea. Rev., 148, 1753-1778, https://doi.org/10.1175/ MWR-D-19-0288.1.

Nowotarski, C. J., and A. A. Jensen, 2013: Classifying proximity soundings with self-organizing maps toward improving supercell and tornado forecasting. Wea. Forecasting, 28, 783801, https://doi.org/10.1175/WAF-D-12-00125.1.

_ , and E. A. Jones, 2018: Multivariate self-organizing map approach to classifying supercell tornado environments using near-storm, low-level wind and thermodynamic profiles. Wea. Forecasting, 33, 661-670, https://doi.org/10.1175/WAFD-17-0189.1.

Orf, L., R. Wilhelmson, B. Lee, C. Finley, and A. Houston, 2017: Evolution of a long-track violent tornado within a simulated supercell. Bull. Amer. Meteor. Soc., 98, 45-68, https://doi.org/ 10.1175/BAMS-D-15-00073.1.

Parker, M. D., 2007: Simulated convective lines with parallel stratiform precipitation. Part II: Governing dynamics and associated sensitivities. J. Atmos. Sci., 64, 289-313, https:// doi.org/10.1175/JAS3854.1.

- 2010: Relationship between system slope and updraft intensity in squall lines. Mon. Wea. Rev., 138, 3572-3578, https:// doi.org/10.1175/2010MWR3441.1.

- 2 2014: Composite VORTEX2 supercell environments from near-storm soundings. Mon. Wea. Rev., 142, 508-529, https:// doi.org/10.1175/MWR-D-13-00167.1.

, 2017: How much does "backing aloft" actually impact a supercell? Wea. Forecasting, 32, 1937-1957, https://doi.org/ 10.1175/WAF-D-17-0064.1.

_, and R. H. Johnson, 2004a: Simulated convective lines with leading precipitation. Part I: Governing dynamics. J. Atmos. Sci., 61, 1637-1655, https://doi.org/10.1175/1520-0469(2004) $061<1637$ :SCLWLP>2.0.CO;2.

$\longrightarrow$, and $-2004 \mathrm{~b}$ : Structures and dynamics of quasi-2D mesoscale convective systems. J. Atmos. Sci., 61, 545-567, https://doi.org/ 10.1175/1520-0469(2004)061<0545:SADOQM>2.0.CO;2.

—_, and J. M. L. Dahl, 2015: Production of near-surface vertical vorticity by idealized downdrafts. Mon. Wea. Rev., 143, 27952816, https://doi.org/10.1175/MWR-D-14-00310.1.

Peters, J. M., C. J. Nowotarski, and H. Morrison, 2019: The role of vertical wind shear in modulating maximum supercell updraft velocities. J. Atmos. Sci., 76, 3169-3189, https://doi.org/ 10.1175/JAS-D-19-0096.1.

Ponmalai, R., and C. Kamath, 2019: Self-organizing maps and their applications to data analysis. LLNL Tech. Rep. lLNL-TR-791165, 51 pp., https://www.osti.gov/servlets/ purl/1566795.

Rasmussen, E. N., and D. O. Blanchard, 1998: A baseline climatology of sounding-derived supercell and tornado forecast parameters. Wea. Forecasting, 13, 1148-1164, https://doi.org/ 10.1175/1520-0434(1998)013<1148:ABCOSD > 2.0.CO;2.

Roberts, B., and M. Xue, 2017: The role of surface drag in mesocyclone intensification leading to tornadogenesis within an idealized supercell simulation. J. Atmos. Sci., 74, 3055-3077, https://doi.org/10.1175/JAS-D-16-0364.1.

,,-- A. D. Schenkman, I. Dawson, and T. Daniel, 2016: The role of surface drag in tornadogenesis within an idealized supercell simulation. J. Atmos. Sci., 73, 3371-3395, https:// doi.org/10.1175/JAS-D-15-0332.1.

— _ - I. Dawson, and T. Daniel, 2020: The effect of surface drag strength on mesocyclone intensification and tornadogenesis in 
idealized supercell simulations. J. Atmos. Sci., 77, 1699-1721, https://doi.org/10.1175/JAS-D-19-0109.1.

Rotunno, R., and J. B. Klemp, 1982: The influence of the shearinduced pressure gradient on thunderstorm motion. Mon. Wea. Rev., 110, 136-151, https://doi.org/10.1175/15200493(1982)110<0136:TIOTSI > 2.0.CO;2.

$\longrightarrow$, and - 1985: On the rotation and propagation of simulated supercell thunderstorms. J. Atmos. Sci., 42, 271-292, https:// doi.org/10.1175/1520-0469(1985)042<0271:OTRAPO >2.0.CO;2.

Schenkman, A. D., M. Xue, and M. Hu, 2014: Tornadogenesis in a high-resolution simulation of the 8 May 2003 Oklahoma City supercell. J. Atmos. Sci., 71, 130-154, https://doi.org/10.1175/ JAS-D-13-073.1.

Sessa, M. F., and R. J. Trapp, 2020: Observed relationship between tornado intensity and pretornadic mesocyclone characteristics. Wea. Forecasting, 35, 1243-1261, https://doi.org/10.1175/ WAF-D-19-0099.1.

Skinner, P. S., C. C. Weiss, M. M. French, H. B. Bluestein, P. M. Markowski, and Y. P. Richardson, 2014: VORTEX2 observations of a low-level mesocyclone with multiple internal rearflank downdraft momentum surges in the 18 May 2010 Dumas, Texas, supercell. Mon. Wea. Rev., 142, 2935-2960, https:// doi.org/10.1175/MWR-D-13-00240.1.

Smith, B. T., R. L. Thompson, J. S. Grams, C. Broyles, and H. E. Brooks, 2012: Convective modes for significant severe thunderstorms in the contiguous United States. Part I: Storm classification and climatology. Wea. Forecasting, 27, 11141135, https://doi.org/10.1175/WAF-D-11-00115.1.

Thompson, R. L., R. Edwards, J. A. Hart, K. L. Elmore, and P. Markowski, 2003: Close proximity soundings within supercell environments obtained from the rapid update cycle. Wea. Forecasting, 18, 1243-1261, https://doi.org/10.1175/15200434(2003)018<1243:CPSWSE $>2.0$. CO;2.

- C. M. Mead, and R. Edwards, 2007: Effective storm-relative helicity and bulk shear in supercell thunderstorm environments. Wea. Forecasting, 22, 102-115, https://doi.org/10.1175/ WAF969.1.

- B. T. Smith, J. S. Grams, A. R. Dean, and C. Broyles, 2012: Convective modes for significant severe thunderstorms in the contiguous United States. Part II: Supercell and QLCS tornado environments. Wea. Forecasting, 27, 1136-1154, https:// doi.org/10.1175/WAF-D-11-00116.1.
Trapp, R. J., 1999: Observations of nontornadic low-level mesocyclones and attendant tornadogenesis failure during VORTEX. Mon. Wea. Rev., 127, 1693-1705, https://oi.org/ 10.1175/1520-0493(1999)127<1693:OONLLM>2.0.CO;2.

_ G. J. Stumpf, and K. L. Manross, 2005: A reassessment of the percentage of tornadic mesocyclones. Wea. Forecasting, 20, 680-687, https://doi.org/10.1175/WAF864.1.

— G. R. Marion, and S. W. Nesbitt, 2017: The regulation of tornado intensity by updraft width. J. Atmos. Sci., 74, 41994211, https://doi.org/10.1175/JAS-D-16-0331.1.

$\longrightarrow, \ldots$, and — 2018: Reply to "Comments on 'The regulation of tornado intensity by updraft width."' J. Atmos. Sci., 75, 4057-4061, https://doi.org/10.1175/JAS-D-18-0276.1.

Vettigli, G., 2019: MiniSom: Self Organizing Maps, version 2.2.3. GitHub, https://github.com/JustGlowing/minisom.

Wakimoto, R. M., and H. Cai, 2000: Analysis of a nontornadic storm during VORTEX 95. Mon. Wea. Rev., 128, 565-592 https:// doi.org/10.1175/1520-0493(2000)128<0565:AOANSD>2.0.CO;2.

Warren, R. A., H. Richter, H. A. Ramsay, S. T. Siems, and M. J. Manton, 2017: Impact of variations in upper-level shear on simulated supercells. Mon. Wea. Rev., 145, 2659-2681, https:// doi.org/10.1175/MWR-D-16-0412.1.

Weisman, M. L., and R. Rotunno, 2000: The use of vertical wind shear versus helicity in interpreting supercell dynamics. J. Atmos. Sci., 57, 1452-1472, https://doi.org/10.1175/15200469(2000)057<1452:TUOVWS > 2.0.CO;2.

Wicker, L. J., and R. B. Wilhelmson, 1995: Simulation and analysis of tornado development and decay within a three-dimensional supercell thunderstorm. J. Atmos. Sci., 52, 2675-2703, https:// doi.org/10.1175/1520-0469(1995)052<2675:SAAOTD > 2.0.CO;2.

Wilhelmson, R., and Y. Ogura, 1972: The pressure perturbation and the numerical modeling of a cloud. J. Atmos. Sci., 29, 1295-1307, https://doi.org/10.1175/1520-0469(1972)029<1295: TPPATN $>2.0 . \mathrm{CO} ; 2$.

Yokota, S., H. Niino, H. Seko, M. Kunii, and H. Yamauchi, 2018: Important factors for tornadogenesis as revealed by highresolution ensemble forecasts of the Tsukuba supercell tornado of 6 May 2012 in Japan. Mon. Wea. Rev., 146, 1109-1132, https://doi.org/10.1175/MWR-D-17-0254.1.

Zeitler, J. W., and M. J. Bunkers, 2005: Operational forecasting of supercell motion: Review and case studies using multiple datasets. Natl. Wea. Dig., 29, 81-97. 